\title{
NF-кB-mediated Pax7 dysregulation in the muscle microenvironment promotes cancer cachexia
}

\author{
Wei A. He, ${ }^{1}$ Emanuele Berardi, ${ }^{2}$ Veronica M. Cardillo, ${ }^{2}$ Swarnali Acharyya, ${ }^{3}$ Paola Aulino, ${ }^{2}$ \\ Jennifer Thomas-Ahner, ${ }^{4}$ Jingxin Wang, ${ }^{1}$ Mark Bloomston, 5 Peter Muscarella, ${ }^{6}$ Peter Nau, ${ }^{6}$ \\ Nilay Shah, ${ }^{6}$ Matthew E.R. Butchbach, ${ }^{7}$ Katherine Ladner, ${ }^{1}$ Sergio Adamo, ${ }^{2}$ Michael A. Rudnicki, ${ }^{8}$ \\ Charles Keller, 9 Dario Coletti, 2 Federica Montanaro,4 and Denis C. Guttridge ${ }^{1,5}$

\begin{abstract}
${ }^{1}$ Human Cancer Genetics Program, Department of Molecular Virology, Immunology and Medical Genetics, The Ohio State University, Columbus, Ohio, USA. 2Department of Anatomical, Histological, Forensic and Orthopaedic Sciences, Rome, Italy. ${ }^{3}$ Cancer Biology and Genetics Program, Memorial Sloan Kettering Cancer Center, New York, New York, USA. ${ }^{4}$ Nationwide Children's Hospital, Columbus, Ohio, USA. ${ }^{5}$ The Arthur G. James Comprehensive Cancer Center, ${ }^{6}$ Department of Surgery, and ${ }^{7}$ Department of Molecular and Cellular Biochemistry, The Ohio State University, Columbus, Ohio, USA. ${ }^{8}$ tttawa Health Research Institute, Ottawa, Ontario, Canada. ${ }^{9}$ Pape Family Pediatrics Research Institute, Oregon Health and Science University, Portland, Oregon, USA.
\end{abstract}

\begin{abstract}
Cachexia is a debilitating condition characterized by extreme skeletal muscle wasting that contributes significantly to morbidity and mortality. Efforts to elucidate the underlying mechanisms of muscle loss have predominantly focused on events intrinsic to the myofiber. In contrast, less regard has been given to potential contributory factors outside the fiber within the muscle microenvironment. In tumor-bearing mice and patients with pancreatic cancer, we found that cachexia was associated with a type of muscle damage resulting in activation of both satellite and nonsatellite muscle progenitor cells. These muscle progenitors committed to a myogenic program, but were inhibited from completing differentiation by an event linked with persistent expression of the self-renewing factor Pax7. Overexpression of Pax7 was sufficient to induce atrophy in normal muscle, while under tumor conditions, the reduction of Pax7 or exogenous addition of its downstream target, MyoD, reversed wasting by restoring cell differentiation and fusion with injured fibers. Furthermore, Pax 7 was induced by serum factors from cachectic mice and patients, in an NF- $\mathrm{kB}$-dependent manner, both in vitro and in vivo. Together, these results suggest that Pax7 responds to NF- $\kappa B$ by impairing the regenerative capacity of myogenic cells in the muscle microenvironment to drive muscle wasting in cancer.
\end{abstract}

\section{Introduction}

Cachexia, a wasting condition associated with chronic illnesses, is primarily characterized by atrophy (wasting) of skeletal muscle that leads to pronounced weight loss (1). In cancer, cachexia patients are at increased risk of adverse outcomes after surgery and chemotherapy (2). Pancreatic and other gastrointestinal cancers present with the highest incidence of cachexia, and one-third of these patients lose $10 \%$ or more of their pre-illness weight $(3,4)$. Sadly, even after decades of research and aggressive treatments, the 5 -year survival rate for pancreatic cancer remains at $6 \%$, among the lowest for all solid tumor malignancies (5). Therefore, efforts to better understand the underlying mechanisms of cachexia may ultimately improve treatment response and quality of life for these and other cancer patients.

Atrophy of skeletal muscle largely derives from aberrant signaling of pathways that maintain a balance between the anabolism and the catabolism of muscle protein. In cachexia, this balance is tipped toward a catabolic state resulting from activated ubiquitin proteasome and autophagy systems that promote protein breakdown as well as from reduced Akt and mTOR activities that decrease protein synthesis (6). Whereas these events are firmly established as residing within the myofiber, less is known regarding the significance of events outside the fiber that might also contribute to muscle wasting in cancer.

Conflict of interest: The authors have declared that no conflict of interest exists. Citation for this article: J Clin Invest. 2013;123(11):4821-4835. doi:10.1172/JCI68523.
The muscle microenvironment includes resident stem cell pools consisting mainly of satellite cells, as well as other interstitial and perivascular populations, that are capable of committing to a myogenic lineage and muscle repair in response to a myotrauma (7). Since the discovery of the satellite cell (8), numerous dynamic processes involving these cells have been associated with various atrophy conditions. In denervation, satellite cell numbers decline, and over time, small new immature fibers form in the interstitial space, potentially resulting from an abortive myogenic program $(9,10)$. In disuse atrophy, the mitotic activity of satellite cells is reduced (11), while in cancer, chronic obstructive pulmonary disease, renal failure, and burn-induced cachexia, the expression of myogenic factors has previously been described (12-15), and in some cases was linked to dysregulated differentiation and muscle loss $(13,16$, 17). However, whether such dynamic changes to satellite cells and myogenesis occur as a consequence of atrophy or are causal for the wasting state is not known. Furthermore, the relevance and contribution of potential events in the muscle microenvironment relative to those mechanisms affecting catabolic processes intrinsic to the myofiber remains to be determined.

Using multiple experimental approaches from murine cancer models and muscle biopsy specimens from cachectic patients, we here describe in detail the regulatory events that occur to satellite cells and, surprisingly, other muscle progenitors. We further describe the unique role of the self-renewing transcription factor Pax7, which, under the control of classical NF-кB signaling, becomes 
A
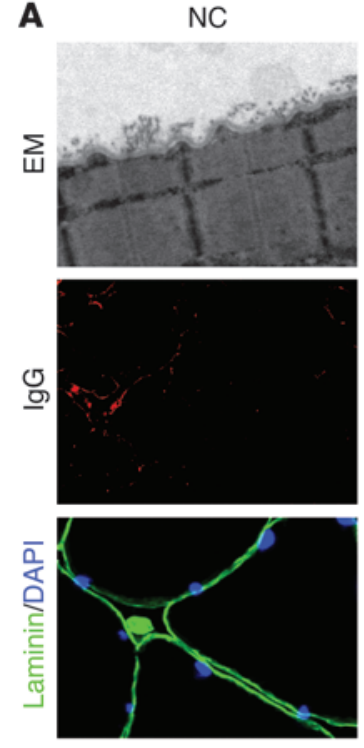

B

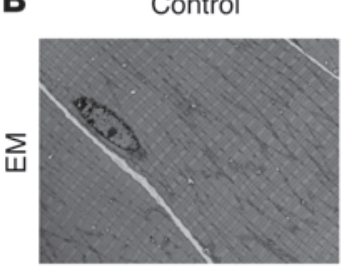

C

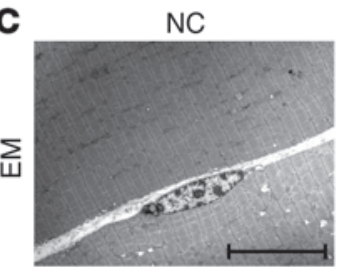

$\mathrm{PC}(0 \%)$
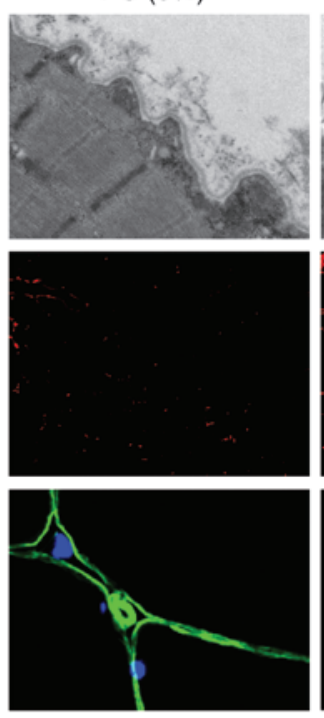

PC (10\%)
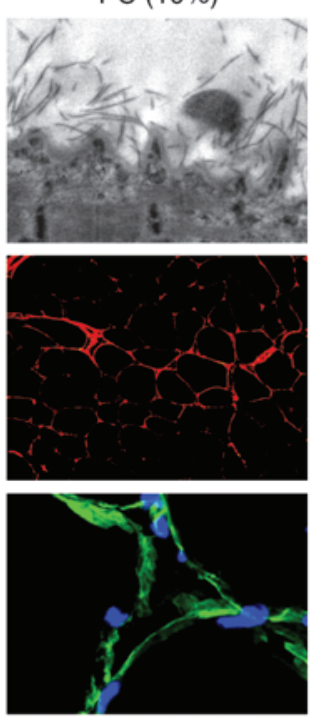

PC (22\%)
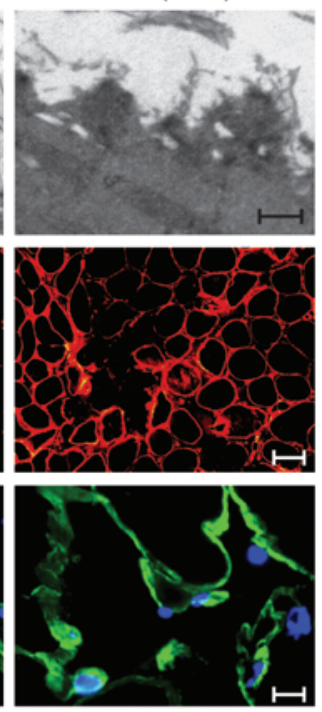

C-26
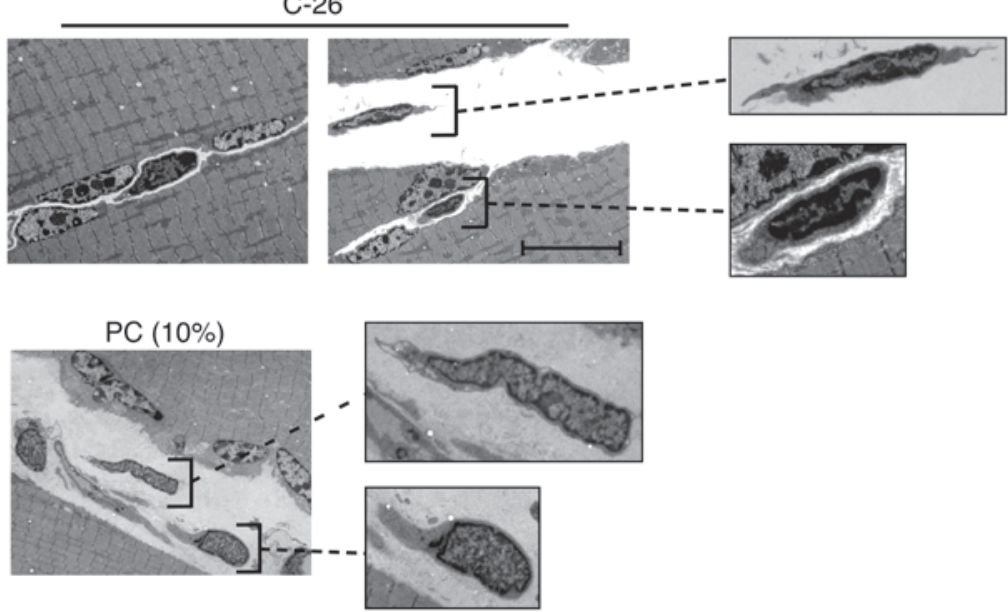

Figure 1

Cancer cachexia promotes myofiber damage and interstitial cell alterations. (A) Top: EM taken from ultrathin longitudinal sections of skeletal muscle biopsies from NC or PC patients. Middle: Human IgG immunofluorescence staining on muscle cross-sections from noncancer individuals (NC) and pancreatic cancer (PC) patients. Bottom: $\alpha$-laminin and DAPI staining on the same patient samples. Percentages in parentheses represent body weight loss compared with pre-illness status. (B) EM analysis of ultrathin muscle longitudinal sections from control and C-26 mice. (C) Sections similar to those in $\mathbf{A}$ were analyzed for cellular alterations. Higher-magnification views of bracketed regions in $\mathbf{B}$ and $\mathbf{C}$ are shown at right (enlarged ×4). Scale bars: $0.5 \mu \mathrm{m}(\mathbf{A}$, top); $100 \mu \mathrm{m}(\mathbf{A}$, middle); $10 \mu \mathrm{m}(\mathbf{A}$, bottom; B; and $\mathbf{C})$.

dysregulated and functions to block myogenic differentiation and promote muscle wasting. Collectively, these findings provide insight into the mechanisms of cachexia by underscoring the importance of events that take place in the muscle microenvironment.

\section{Results}

Cancer cachexia is clinically associated with muscle damage and satellite cell activation. Previous histological analysis of skeletal muscles from tumor-bearing Colon-26 (C-26) mice suggested that this model faithfully recapitulates the clinical features of cancer-induced muscle wasting (18). Similar to the human condition, cachexia in the C-26 model results from the atrophy of type II fibers, but signs of infiltrating immune cells (which are more typical of muscular dystrophies) are absent (19). Yet as with muscular dystrophy, myo- fibers from C-26 mice exhibit alteration to the sarcolemma and basal lamina resembling a damage-like phenotype (18). Because muscle damage triggers satellite cell activation (20), we set out to test whether muscle injury occurs in cancer. Hindlimb muscles from C-26 mice contained a pronounced accumulation of IgG, used as a marker of membrane damage (21). Alteration in IgG correlated with diffuse laminin staining, increased penetration of Evans blue dye (used as a second marker of membrane damage), and reduced expression of extracellular matrix genes (Supplemental Figure 1, A-C; supplemental material available online with this article; doi:10.1172/JCI68523DS1). These data supported the notion that the sarcolemma and basal lamina are perturbed in cachectic muscle. However, these perturbations were not due to the spreading of tumor cells, since imaging combined with ex vivo 

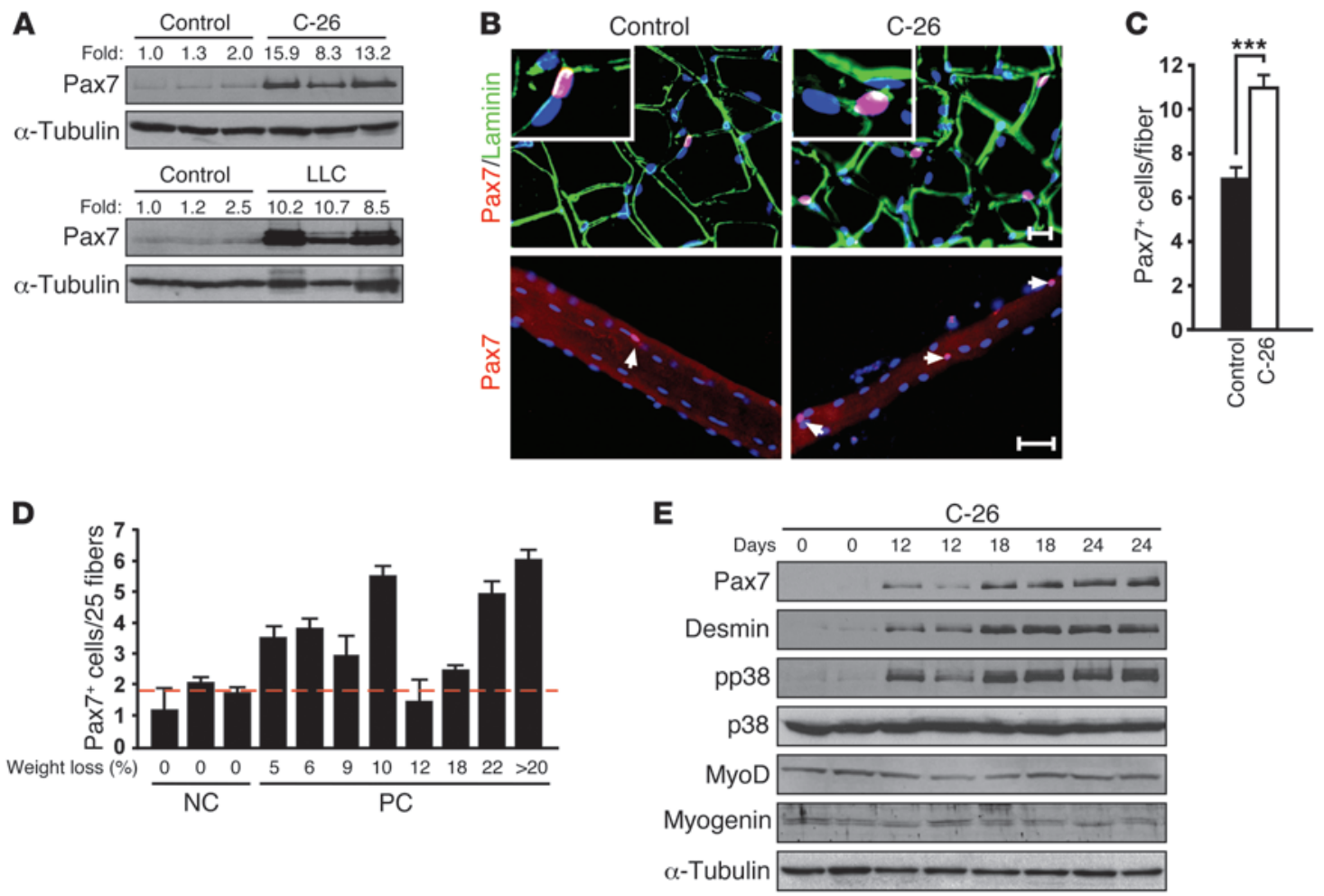

$\mathbf{F}$

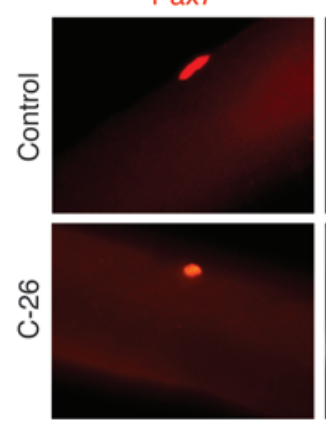

BrdU
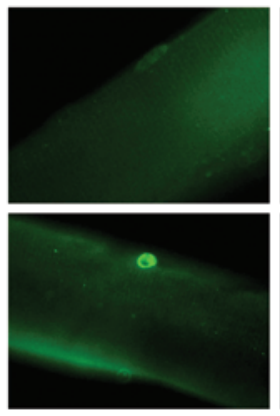
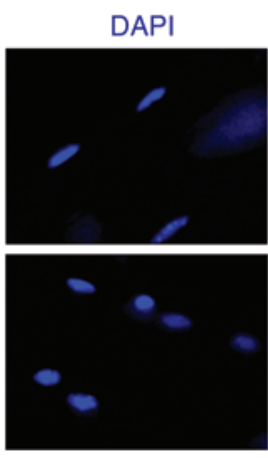

Merge

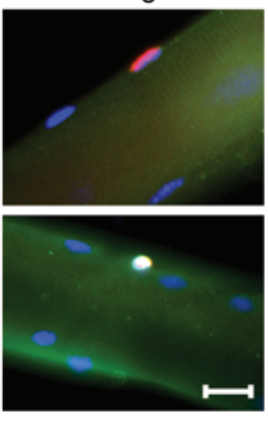

G

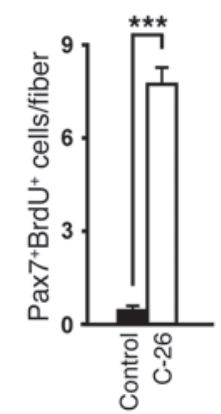

Figure 2

Cancer cachexia is associated with activation and myogenic commitment of satellite cells. (A) GAST from control and C-26 and LLC tumor-bearing mice blotted for Pax7. Numbers above lanes represent Pax7 quantitation by ImageJ, normalized to $\alpha$-tubulin. (B) Top: GAST sections probed for Pax7 and $\alpha$-laminin. Similar results were observed in TA and QUAD. DAPI was used to counterstain nuclei (blue). Insets show $1 \mathrm{Pax} 7{ }^{+}$cell each at higher magnification (enlarged $\times 9$ ). Bottom: Isolated myofibers probed for Pax7 and DAPI (blue). Arrows denote Pax $7^{+}$cells. (C) Quantitation of Pax $7^{+}$cells from single fibers from control and C-26 mice. (D) Cross-sections of muscle biopsies from NC and PC patients, with weight loss as indicated, scored for Pax $7^{+}$cells. Quantitation was performed from 20 random fields, counting a minimum of 1,000 fibers per sample. Dashed red line denotes the baseline value of Pax $7^{+}$cells in NC patients. (E) Muscles were harvested in duplicate experiments after C-26 administration. Western blots were performed, probing for markers of satellite cells (Pax7), myoblasts (phospho- and total p38, desmin, and MyoD), and differentiation (MyoD and myogenin). Blot was reprobed for $\alpha$-tubulin. (F) Single fibers were double stained for Pax7 and BrdU. Nuclei were counterstained with DAPI. (G) Quantitation of Pax7+BrdU+ cells in F. Scale bars: $10 \mu \mathrm{m}$ (B, top); $50 \mu \mathrm{m}$ (B, bottom); $20 \mu \mathrm{m}(\mathbf{F}) .{ }^{* * *} P<0.001$.

luminometry showed that tumors remained localized to the injection site (Supplemental Figure 1, D and E). This suggested that the damage phenotype derives from circulating factors, rather than from tumor-infiltrating cells within the muscle fascia.

To determine the clinical relevance of this muscle damage response, a similar analysis was repeated with muscle biopsies from patients with pancreatic cancer that exhibited varying degrees of weight loss (PC patients; Supplemental Table 1). EM demonstrated an ordered appearance of myofiber sarcolemma from weight-stable, noncancer individuals (NC patients; Figure 1A). In contrast, those from PC patients exhibited membrane alterations that worsened with increasing weight loss. Muscles of PC patients also exhibited elevated IgG and diffuse laminin staining (Figure 1A). In general, the greatest ultrastructural changes contained the highest IgG accumulation and laminin disruption, correlating positively with cachexia severity. Our observations corroborated the C-26 
A

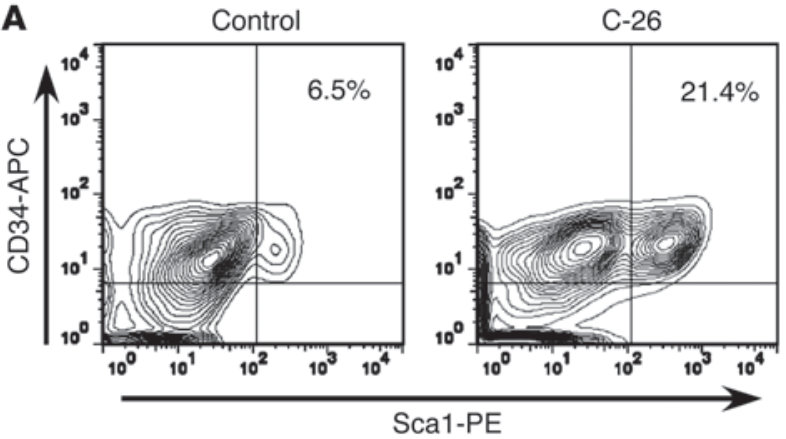

B

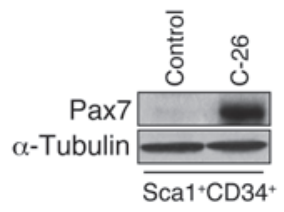

C

$\operatorname{Pax} 7$

Sca1

DAPI
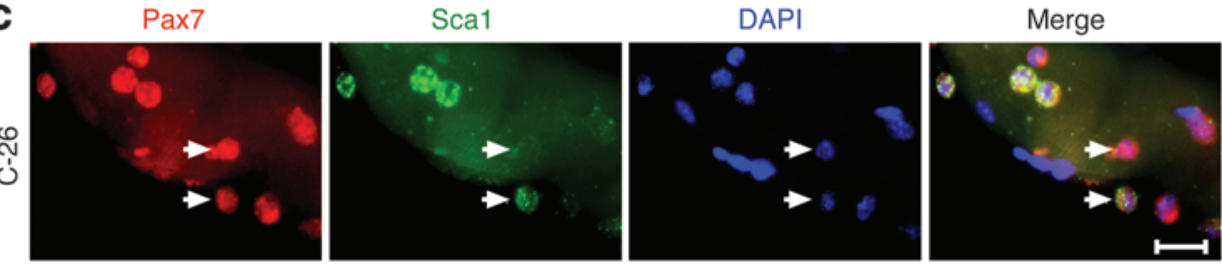

D

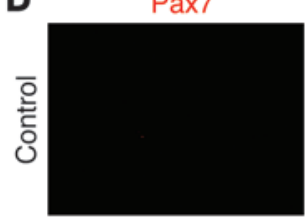

Sca1
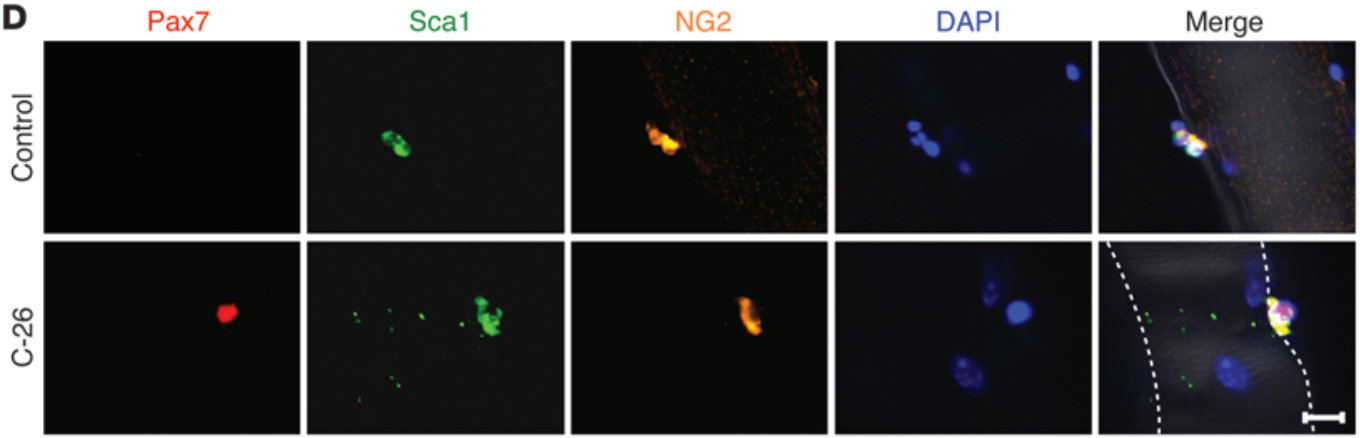

$\operatorname{Pax} 7$
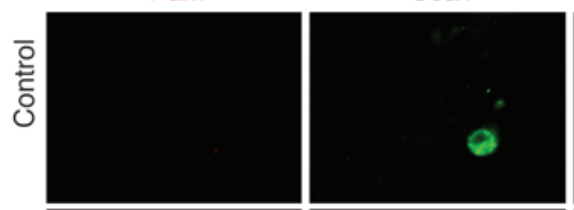

PDGFRQ
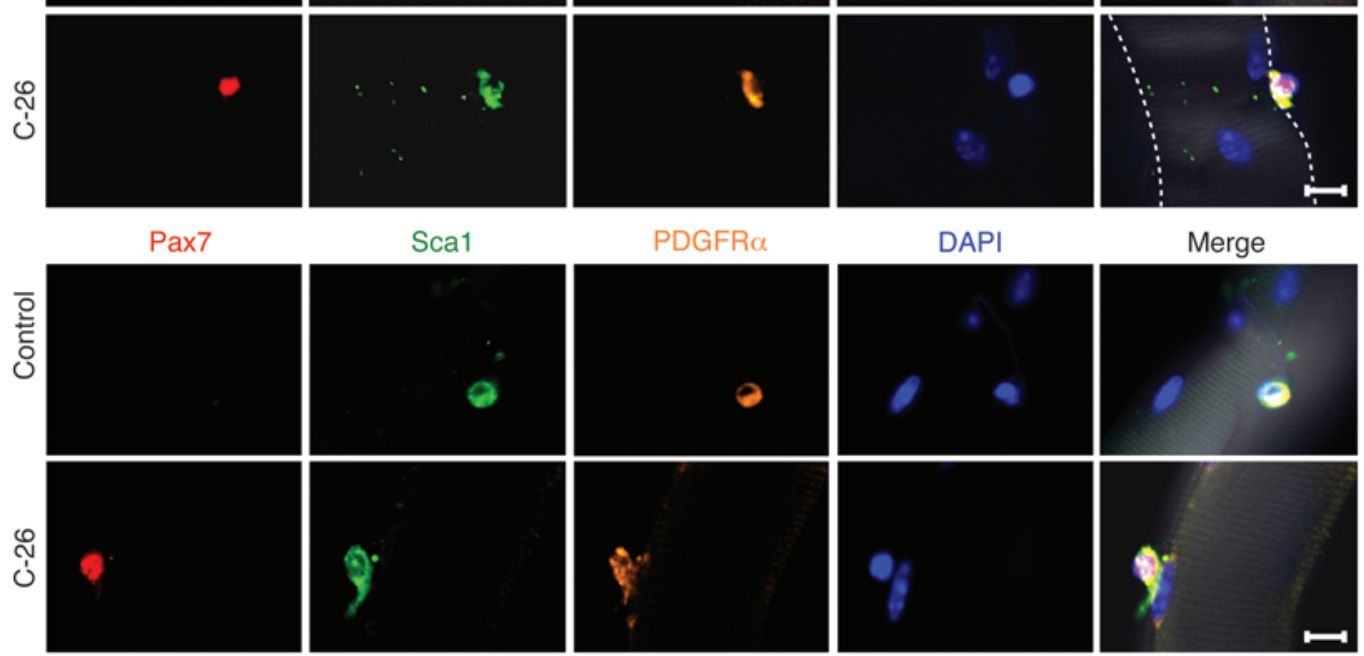

E

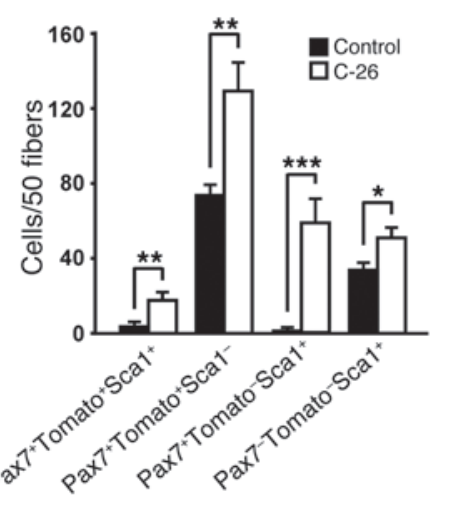

$\mathbf{F}$

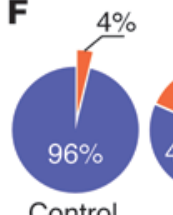

Control
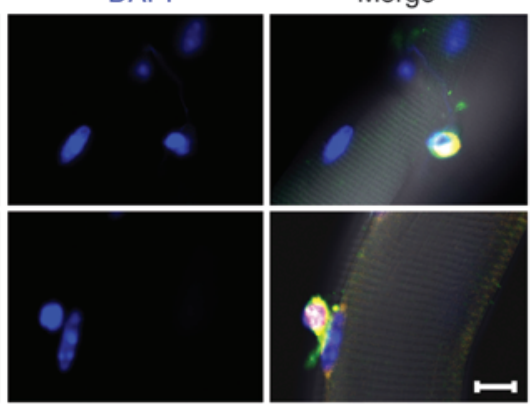

Pax7 $7^{+}$Tomato-Sca1+ Pax7-Tomato-Sca1+

\section{Figure 3}

Cancer cachexia is associated with myogenic commitment of multiple muscle progenitor cells. (A) FACS analysis of nonsatellite progenitor cells in control and C-26 mice by gating for Sca $1^{+} \mathrm{CD} 34^{+}$cells (shown as a percentage). (B) Cells as in A were sorted and probed for Pax7 by Western blot. (C) Single fibers from C-26 mice were stained with Pax7 and Sca1. Nuclei were counterstained with DAPI. Arrows denote Pax $7^{+} \mathrm{Sca} 1^{-}$and $\mathrm{Pax} 7^{+} \mathrm{Sca} 1^{+}$ cells. (D) Single fibers from control and C-26 mice were immunostained with Pax7, Sca1, NG2 or PDGFR $\alpha$, and DAPI as indicated. Dotted white lines indicate myofiber border. (E) Myofibers were isolated from Pax7-Cre ${ }^{E R}$;Rosa26Tomato reporter control and C-26 mice that were induced with tamoxifen before tumor implantation, and myofibers were subsequently immunostained for Pax7 and Sca1. For each condition, at least 300 fibers were counted. Quantitation of 4 cell populations is shown. The major increase of Pax ${ }^{+}$Sca1+ cells came from a nonsatellite cell lineage. (F) Distribution of Pax7 expression in the Tomato-Sca1+ population of control and C-26 mice. Scale bars: $20 \mu \mathrm{m}(\mathbf{C}) ; 25 \mu \mathrm{m}(\mathrm{D}) .{ }^{*} P<0.05,{ }^{\star \star} P<0.01,{ }^{* \star} P<0.001$. 
model, and together the data established that cachexia is clinically associated with tumor-induced muscle damage.

Interestingly, concomitant with the damage phenotype, muscles from tumor-bearing mice and PC patients contained an accumulating number of cells in the interstitium that appeared morphologically distinct from myonuclei with respect to their heterochromatin and extension of their cytoplasm (Figure 1, B and C). Such markings are characteristic of activated satellite cells, which normally are anatomically positioned between the myofiber sarcolemma and basal lamina, but upon muscle injury exit from quiescence and subsequently expand as myoblasts to repair damaged fibers (22). To assess the identity of these cells, we probed for the satellite cell marker Pax7 (23) and observed reproducibly higher Pax7 expression in gastrocnemius (GAST), tibialis anterior (TA), and quadriceps (QUAD) from C-26 mice compared with saline-injected controls (Figure 2A and data not shown). This was not specific to C-26 tumors, as Pax7 was also elevated in a second cachexia model generated from Lewis lung carcinoma (LLC) cells (Figure 2A and ref. 18). Immunostaining of muscle sections revealed an expansion of $\mathrm{Pax}^{+}$cells that resided mostly outside the basal lamina (Figure $2 \mathrm{~B}$ ) and correlated with EM findings. These results were supported by quantitation on single fibers, where we observed that cachectic fibers contained $60.3 \% \pm 8.0 \%$ more $\mathrm{Pax} 7^{+}$cells than controls $\left(P=0.0002\right.$; Figure 2, B and C). Notably, $\mathrm{Pax} 7^{+}$cells were also significantly increased in biopsies from PC patients $(P<0.01$; Figure $2 \mathrm{D}$ and Supplemental Figure 2A). Together, these data indicated that cancer cachexia associates with an expansion of satellite cells.

In line with these findings, Pax7 from total muscle homogenate was induced 12 days after C-26 tumor administration (Figure $2 \mathrm{E}$ ), a time that precedes the onset of muscle atrophy (18). Pax7 also steadily increased during cachexia progression, which mirrored expression of the satellite cell activation markers desmin and phospho-p38 $\alpha$. To analyze whether satellite cells expand in cancer, myofibers were isolated from mice treated with the thymidine analog BrdU. These demonstrated a prominent increase in $\mathrm{Pax}^{+} \mathrm{BrdU}^{+}$satellite cells that were tightly associated with cachectic myofibers (Figure 2, F and G). Further staining revealed coexpression of Pax7 with desmin as well as with a second proliferation marker, phospho-histone H3 (Supplemental Figure 2, B and C). Together, these data suggest that circulating cachectic factors induce muscle damage and activation of satellite cells at an early stage of cachexia development, and that cells in the interstitium likely reflect activated rather than quiescent satellite cells that have detached from the fiber due to membrane damage.

Cancer cachexia induces myogenic commitment of nonsatellite progenitor cells. Although significant, the extent to which satellite cells expanded in cancer cachexia did not reflect the more than 10-fold higher levels of Pax7 measured in cachectic muscles $(P=0.03$; Figure 2A). This raised the intriguing possibility that another cell population may initiate Pax7 expression in tumor-induced muscle damage. Multiple progenitors within the muscle interstitium adopt a myogenic lineage in response to injury (24-26). Although little is known about the origins of these populations, they nevertheless share the cell surface markers Sca1 and CD34 (27). Therefore, we asked whether cells positive for these markers are also regulated in cachexia. Fluorescence-activated cell sorting (FACS) of mononuclear cells revealed pronounced expansion of $\mathrm{Sca} 1^{+} \mathrm{CD} 34^{+}$cells in cachectic muscle (Figure 3A). Importantly, Pax7 was strongly associated with purified Sca $1^{+} \mathrm{CD} 34^{+}$cells (Figure $3 \mathrm{~B}$ ), which indicated that at least a portion of these cells committed to a myogenic lineage. This notion was substantiated by immunostaining of single myofibers: $\mathrm{Pax} 7^{+} \mathrm{Sca} 1^{+}$cells were clearly distinguishable from $\mathrm{Pax}^{+} \mathrm{Sca} 1^{-}$satellite cells (Figure $3 \mathrm{C}$ and Supplemental Figure 2D). RT-PCR analysis further revealed that the FACS-isolated $\mathrm{Sca} 1^{+} \mathrm{CD} 34^{+}$fraction included cell populations that give rise to myogenic cells, namely, NG2-expressing mesoangioblasts/pericytes and PW1-expressing interstitial cells (PICs) (Supplemental Figure 3A and ref. 25). Whereas $80.3 \%$ of $\mathrm{Sca} 1^{+} \mathrm{NG} 2^{+}$cells were prominently associated with Pax7 in cachectic fibers, $<2 \%$ were observed in control muscle (Figure $3 \mathrm{D}$ and Supplemental Figure 3B). Similar results were observed from LLC tumor-bearing mice (Supplemental Figure 3C). Moreover, $\mathrm{Pax}^{+} \mathrm{Sca} 1^{+} \mathrm{NG} 2^{+}$cells stained positive for phospho-histone $\mathrm{H} 3$ and BrdU (Supplemental Figure 3, D and E), which suggests that - similar to satellite cells - these cells also expand under tumor conditions, potentially representing a subfraction of cells that accumulate in the interstitium during cachexia.

Interestingly, additional immunostaining revealed coexpression of the mesenchymal marker PDGFR $\alpha$ in proliferating $\mathrm{Pax} 7^{+} \mathrm{Sca} 1^{+}$ cells, which were found to be contained in the Sca $1^{+} \mathrm{CD} 34^{+}$population by RT-PCR and immunohistochemical analyses (Figure 3D and Supplemental Figure 3, A-C, and F). PDGFR $\alpha^{+}$progenitors are considered distinct from satellite cells and nonmyogenic, but contribute to muscle repair through expression of trophic factors $(28,29)$. Because such cells have yet to be examined in the context of cancer, it is possible that mesenchymal progenitors enter a myogenic lineage in response to chronic muscle injury induced by tumor progression. Gene expression profiling using NanoString technology verified that FACS-sorted $\mathrm{Sca} 1^{+} \mathrm{CD} 34^{+}$cells from cachectic muscle contained elevated levels of both interstitial (NG2, PW1, and PDGFR $\alpha$ ) and satellite cell (Pax7, integrin $\alpha 7, c-m e t$, and myf5) markers (Supplemental Figure 3G). However, stromal markers characteristic of vascular progenitors, adipocytes, fibroblasts, and osteoblasts were not similarly affected, which indicated that $\mathrm{Sca} 1^{+} \mathrm{CD} 34^{+}$cells in cachexia become preferentially committed to a myogenic lineage.

Since satellite cells are heterogeneous and in some cases express Sca1 (30-32), we performed lineage tracing using Pax7-Cre ${ }^{E R}$;Rosa26Tomato mice (33) to determine whether $\mathrm{Pax} 7^{+} \mathrm{Sca} 1^{+}$cells in cachectic muscle originate from a satellite cell population. In these mice, exposure to tamoxifen leads to Cre-mediated expression of the Tomato reporter in cells and their progeny expressing Pax7. Tamoxifen was administered to mice prior to tumor implantation, because Sca $1^{+} \mathrm{CD} 34^{+}$interstitial cells do not express Pax7 at this stage, so only satellite cells would be marked by Tomato. Therefore, after tumor implantation, $\mathrm{Pax} 7^{+}$cells derived from satellite cells remain Tomato $^{+}$, while those arising from $\mathrm{Sca} 1^{+} \mathrm{CD} 34^{+}$interstitial cells are TomatoIn nontumor mice, nearly $100 \%$ of $\mathrm{Pax}^{+}$cells were Tomato $^{+}$(Supplemental Figure 4A). The large majority of these cells (>90\%) were Sca1-, but, as reported previously $(30,31)$, a rare population of $\mathrm{Pax}^{+}{ }^{+} \mathrm{Tomato}^{+} \mathrm{Sca} 1^{+}$cells was also seen (Figure $3 \mathrm{E}$ and Supplemental Figure 4B). In contrast, tumor mice contained an increase in Pax7Tomato-Sca ${ }^{+}$stem cells, but this did not compare with the dramatic, $>40$-fold expansion of $\mathrm{Pax}^{+}{ }^{+} \mathrm{Tomato}^{-} \mathrm{Sca} 1^{+}$cells, clearly distinct from $\mathrm{Pax}^{+} \mathrm{Tomato}^{+}$satellite cells $(P<0.001$; Figure $3 \mathrm{E}$ and Supplemental Figure 4, A-C). Cumulatively, our results showed that de novo expression of Pax7 occurred in $>50 \%$ of Tomato $^{-} \mathrm{Sca}^{+}$nonsatellite cells (Figure 3F). To account for the possibility that $\mathrm{Pax}^{+}{ }^{+}$Tomato $^{-}$ cells represent satellite cells in which Tomato expression is absent due to inefficient Cre recombinase activity, we further stained for 
A
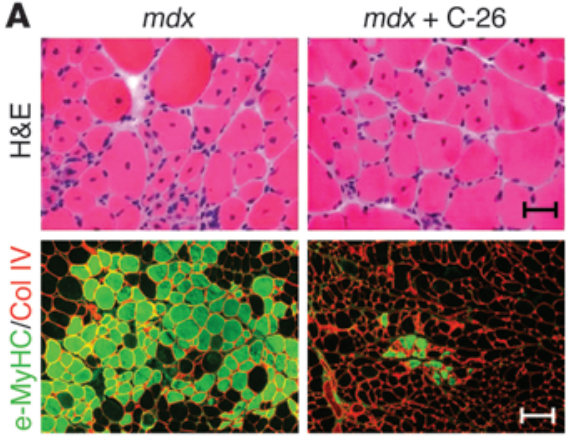

D

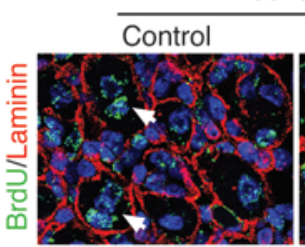

$\mathbf{F}$

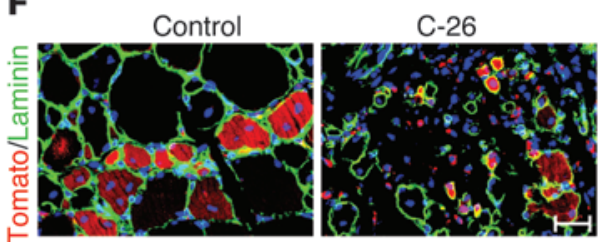

B

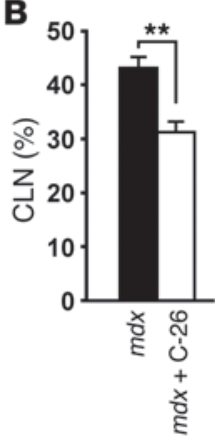

C

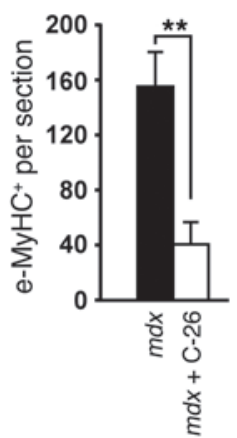

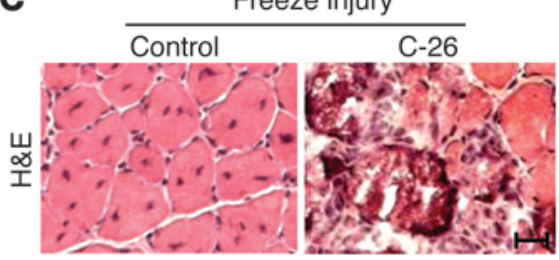

E
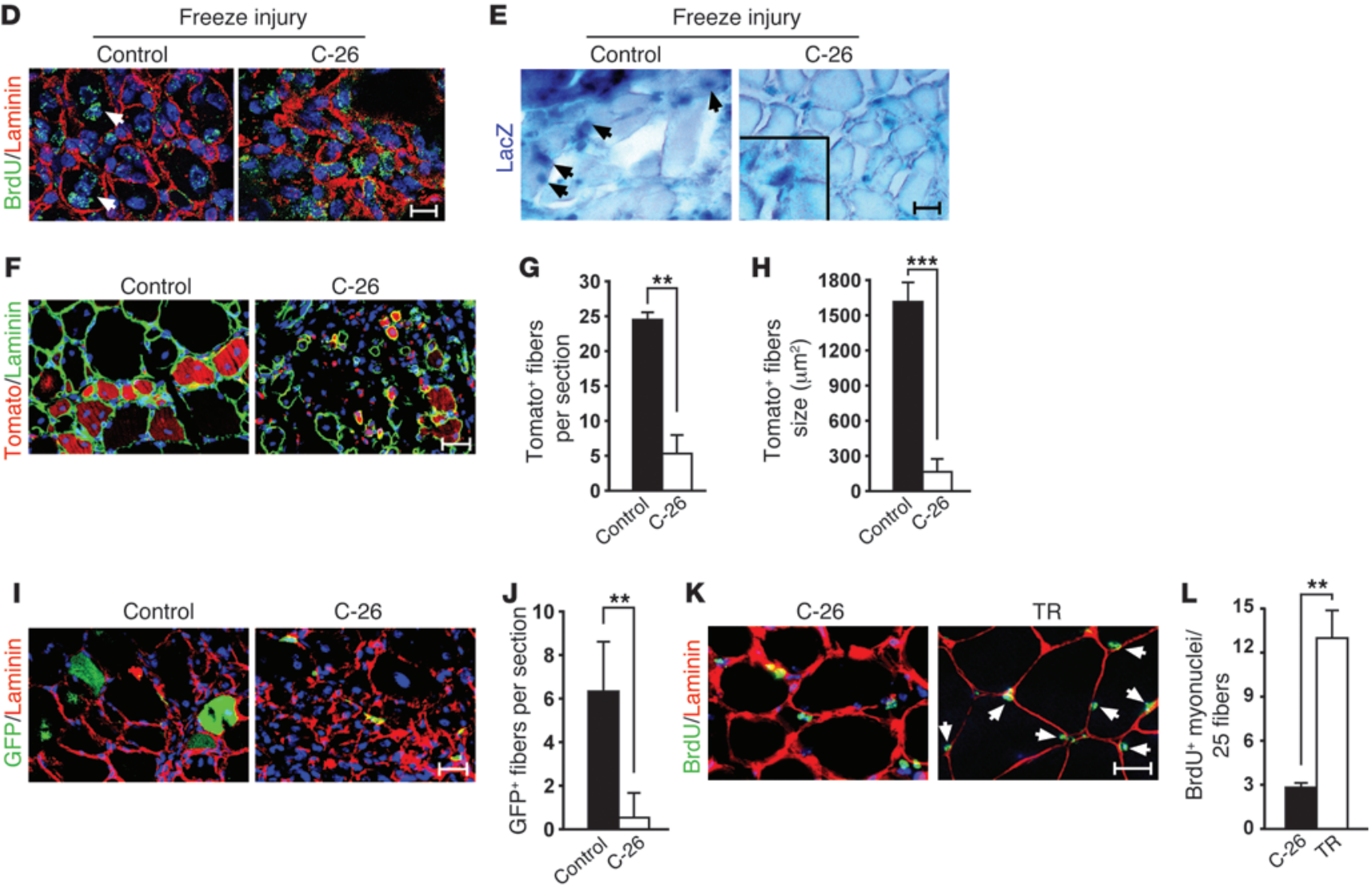

Figure 4

Muscle regeneration is compromised in cancer cachexia. (A and B) 6-week-old $m d x$ mice were injected with C-26 tumors or saline. (A) After 2 weeks, GAST were analyzed by H\&E or immunostained with e-MyHC and collagen IV (Col IV). (B) Quantitation of centrally located nuclei (CLN) and e-MyHC+ fibers. (C) TA from control and C-26 mice were injured by freezing and analyzed after 8 days by H\&E. (D) TA were injured, and mice were subsequently injected with BrdU. TA was immunostained with BrdU and $\alpha$-laminin. (E) Athymic nude control and C-26 mice were subjected to TA injury and transplanted with muscle mononuclear cells from desmin-nLacZ transgenic mice. 1 week after injury, TA was stained for LacZ. Arrows denote desmin ${ }^{+}$nuclei within myofibers. Inset shows interstitial LacZ ${ }^{+}$cells (enlarged $\left.\times 5\right)$. $(\mathbf{F}-\mathbf{H})$ TA from control $(n=5)$ and C-26 $(n=7)$ mice were injured and transplanted with FACS-sorted Tomato cells. (F) After transplantation, muscle sections were immunostained for $\alpha$-laminin and DAPI (blue). Tomato myofiber number $(\mathbf{G})$ and size $(\mathbf{H})$ were also quantified. (I and $\mathbf{J})$ As in $\mathbf{F}$, except that the donor population was sorted for $\mathrm{CD}_{34}{ }^{+}$Sca $1^{+}$cells from nontumor GFP reporter mice. (I) Immunostaining of GFP, $\alpha$-laminin, and DAPI (blue). (J) GFP+ myofibers. (K and L) C-26 mice and surgically tumor-resected C-26 mice (TR) were injected with BrdU ( $n=5$ per group). (K) Sections were stained for BrdU, $\alpha$-laminin, and DAPI (blue). (L) Sublaminar BrdU+ nuclei from tumor-resected animals. Scale bars: $25 \mu \mathrm{m}(\mathbf{A}, \mathrm{H} \& \mathrm{E}) ; 100 \mu \mathrm{m}(\mathbf{A}$, immunostaining); $20 \mu \mathrm{m}(\mathbf{C}$; E; F; I; and K); $10 \mu \mathrm{m}(\mathbf{D}) .{ }^{* *} P<0.01,{ }^{* * *} P<0.001$.

NG2 and PDGFR $\alpha$, which would not be expected to mark satellite cells. In cachectic fibers, Pax $7^{+}$Tomato ${ }^{-}$cells also expressed NG2 or PDGFR $\alpha$ (Supplemental Figure 4D), which excluded the possibility that $\mathrm{Pax}^{+}{ }^{+}$Tomato $^{-}$cells arise from satellite cells in tumor-bearing mice. In addition, no de novo expression of NG2 or PDGFR $\alpha$ was observed on $\mathrm{Pax}^{+} \mathrm{T}$ mato $^{+}$satellite cells (Supplemental Figure 4E). Together, these data reveal that cancer-induced muscle wasting promotes the myogenic activation of multiple stem cell populations. 
Wasting in cachexia is linked with aberrant progenitor cell activation and differentiation. Given that expression of NG2 and PDGFR $\alpha$ is absent in quiescent and activated myogenic cells $(28,29,34)$, our findings indicated that commitment of nonsatellite cell progenitors might be perturbed under tumor conditions. To explore whether a similar phenomenon occurs with satellite cells, we examined expression of CD34, a marker that is rapidly downregulated upon satellite cell activation and commitment to myogenesis $(35,36)$. Cachectic mice contained greater than twice the number of $\mathrm{Pax} 7{ }^{+} \mathrm{CD} 34^{+}$cells compared with controls ( $P=0.006$; Supplemental Figure $5 A)$, and FACS confirmed the expansion of $\mathrm{CD} 34^{+}$satellite cells in cachectic muscle identified as integrin- $\alpha 7^{+} \mathrm{CD} 31^{-} \mathrm{CD} 45^{-} \mathrm{CD} 11 \mathrm{~b}^{-} \mathrm{Sca} 1^{-}$(Supplemental Figure $5 \mathrm{~B}$ ). Thus, activated satellite cells in tumor-bearing mice retain $\mathrm{CD} 34$, which together with our other results imply that cancer factors induce aberrant activation of both satellite and nonsatellite interstitial progenitors.

To investigate whether this aberrant activation leads to dysregulation of the myogenic program, we probed for expression of MyoD and myogenin, 2 transcription factors downstream of Pax7 that sequentially drive the differentiation of myogenic cells and their fusion into myofibers (37-39). In tumor-bearing mice, unlike the observed increase of phospho-p38 $\alpha$ and desmin, similar induction of MyoD or its transcriptional target, myogenin (35), was not obtained, as shown by blotting of whole muscle extracts and by mRNA and protein analysis of FACS-sorted satellite and $\mathrm{Sca}^{+} \mathrm{CD} 34^{+}$cells (Figure 2E, Supplemental Figure 3G, and Supplemental Figure 5, C and D). Because MyoD and myogenin are essential mediators of differentiation (37-39), their deregulation could lead to impaired regeneration. Therefore, we tested the effects of a tumor on muscle regeneration using in vivo models, in which newly generated myofibers were scored by the presence of centrally located nuclei and expression of embryonic myosin heavy chain (e-MyHC) (37-39). Using the $m d x$ mouse model of Duchenne muscular dystrophy (see Methods), whose muscles undergo repeated cycles of degeneration and regeneration due to a mutation in the dystrophin gene (40), we found that muscles of tumor-free $m d x$ mice contained the expected high number of regenerating fibers (Figure $4, \mathrm{~A}$ and B). In contrast, $m d x$ mice bearing C-26 tumors had a pronounced reduction in myofibers with central nuclei $(27.6 \%$; $P=0.001)$ and e-MyHC (74.1\%; $P=0.004)$. Similar results were obtained from LLC tumor-bearing $m d x$ mice (Supplemental Figure $6 \mathrm{~A})$. Likewise, in an acute model of muscle damage in which regeneration occurs synchronously over a large muscle area, new fibers with central nuclei were abundant in postinjury control muscles. In comparison, postinjured muscles from cachectic mice had numerous patches of necrotic fibers surrounded by inflammatory cells that correlated with a quantitative reduction in myofiber size (Figure 4C and Supplemental Figure 6B). These results demonstrated that tumor factors impair muscle regeneration.

To identify the affected stage of regeneration, we monitored the fate of activated myogenic cells. During normal regeneration, $\operatorname{Pax} 7^{+}$satellite cells proliferate and give rise to myoblasts that subsequently fuse with each other or with preexisting myofibers (37-39). We therefore tested the incorporation of newly fused nuclei into myofibers by labeling proliferating cells with BrdU and tracking their localization in acutely injured muscles. In control mice, $\mathrm{BrdU}^{+}$ nuclei localized to a sublaminar position $\left(675 \pm 175\right.$ nuclei $\left./ \mathrm{mm}^{2}\right)$, signifying successful regeneration. However, in C-26 mice, BrdU ${ }^{+}$ nuclei predominantly resided in the muscle interstitium, with fewer in a sublaminar position $\left(100 \pm 25\right.$ nuclei $/ \mathrm{mm}^{2} ; P<0.05$;
Figure 4D), indicative of a myoblast fusion defect. To determine whether cells that failed to fuse represented myoblasts, we transplanted genetically marked mononuclear cells from desmin-nLacZ transgenic mice (41) into acutely injured muscles of control and tumor-bearing athymic nude mice. In control mice, the expected localization of $\mathrm{LacZ}^{+}$nuclei within myofibers was obtained 1 week after injury, and in some cases, $\mathrm{LacZ}^{+}$nuclei were centrally positioned (Figure 4E). In sharp contrast, muscle sections from C-26 mice clearly showed $\mathrm{LacZ}^{+}$myoblasts remaining in the interstitial space. Together, these results suggested that impaired regeneration in tumor-bearing mice is linked to a myoblast fusion defect.

To determine whether fusion-defective myoblasts derived from satellite as well as nonsatellite progenitor cells, transplantation experiments were performed with purified cells marked with different reporters. For satellite cells, FACS-isolated Tomato ${ }^{+}$cells from tamoxifen-treated Pax7-Cre ${ }^{E R}$;Rosa26-Tomato mice were transplanted into acutely injured TA muscles of control and C-26 mice. At 1 week after transplantation, Tomato ${ }^{+}$fibers were observed in control mice, indicative of successful myoblast fusion, whereas in $\mathrm{C}-26$ mice, Tomato $^{+}$cells remained mainly outside the fiber (Figure 4, F-H). An identical procedure was repeated for nonsatellite cells from GFP reporter mice sorted for CD34 and Sca1. Analogous to satellite cells, $\mathrm{CD}^{2} 4^{+} \mathrm{Sca} 1^{+} \mathrm{GFP}^{+}$cells gave rise to $\mathrm{GFP}^{+}$fibers under control, but not tumor, conditions (Figure 4, I and J). Collectively, these data suggest that regeneration is compromised in cancer due to a fusion defect from potentially multiple myogenic populations.

To address whether this tumor-induced impairment in myoblast differentiation was a permanent event, isolated mononuclear cells were cultured directly in differentiation medium without prior expansion. Compared with controls, cells isolated from tumorbearing mice showed a greater fusion index, with a quantitatively higher percentage of $\mathrm{MyHC}^{+}$myotubes (Supplemental Figure 6, $\mathrm{C}$ and D). This indicates that myogenic cells exposed to cachectic factors retain the capacity to differentiate, and, once removed from their tumor environment, differentiate with accelerated kinetics due to their induced activation state. To address whether this process is recapitulated in vivo, C-26 tumors were surgically removed, and BrdU was injected to track the fate of activated myoblasts. Tumor resection led to a 3-fold increase in myoblast fusion $(P=0.002$; Figure 4, $\mathrm{K}$ and $\mathrm{L})$. Moreover, this event was accompanied by a $25 \%$ increase in fiber size $(P=0.002$; Supplemental Figure $6 \mathrm{E})$. These results demonstrated that inhibition of regeneration in cachectic muscles is a reversible process, which may have important implications from a therapeutic standpoint.

Dysregulation of Pax 7 causes muscle wasting. Next, we determined the mechanism underlying the block in myoblast differentiation. We turned our attention to Pax7, given that this protein was constitutively expressed in cachectic muscle (Figure 2A) and was previously shown to inhibit myogenic differentiation when upregulated, at least in cultured cells (42). A gain-of-function approach was used to test whether overexpression of Pax7 affects muscle mass in vivo. Hindlimb muscles from C-26 mice were infected with a Pax7expressing adeno-associated virus (AAV-Pax7). Compared with muscles infected with control AAV-GFP, Pax7-expressing muscles were significantly reduced in mass (GAST, 28.7\% reduction; TA, $51.9 \%$ reduction; $P<0.0001$ ) and size (GAST, $22.6 \%$ reduction; $P=0.001$ ) (Supplemental Figure 7, A and B). This illustrated that maintaining Pax7 levels, in the context of a tumor, enhances muscle wasting. To determine whether Pax7 alone is sufficient to induce atrophy, we next took advantage of the natural pro- 
A

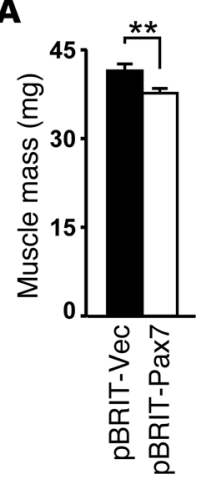

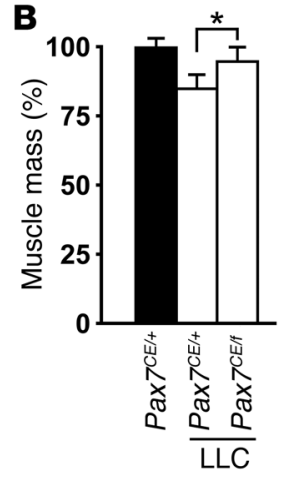

E

口Pax7-Cre ${ }^{E R}, D T A$

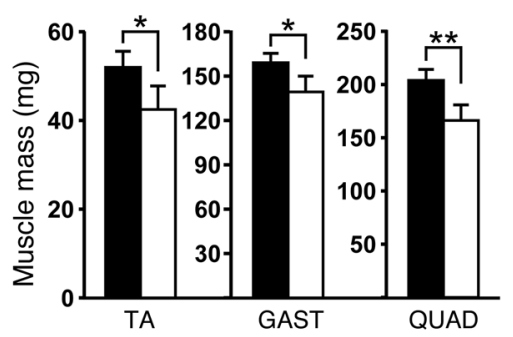

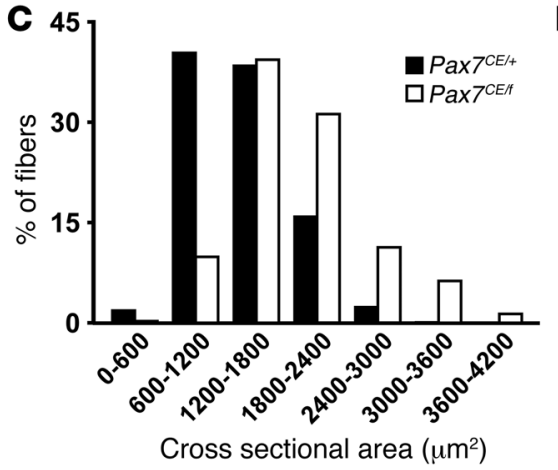

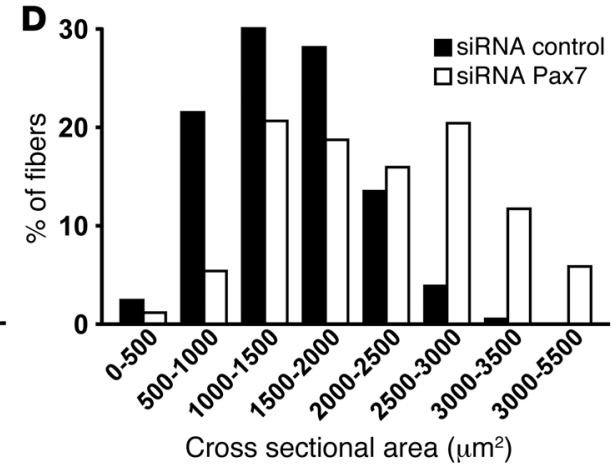

$\mathbf{F}$

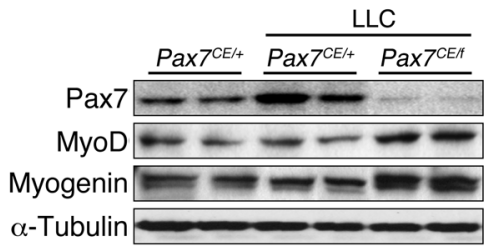

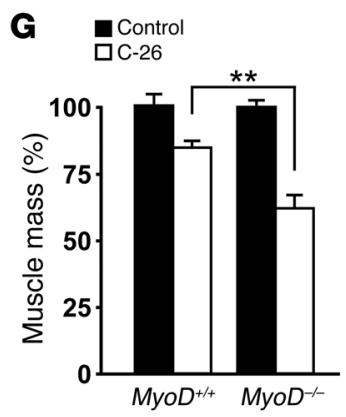

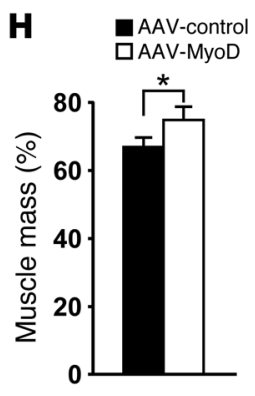

Figure 5

Muscle wasting in cancer cachexia is mediated through deregulation of Pax7. (A) GAST mass, recorded in young mice (P15) after injection of pBRIT retroviruses expressing empty vector $(\mathrm{Vec})$ or the Pax7 transgene. (B) GAST mass from LLC tumor-bearing Pax7CE/+ and Pax7CE/f mice ( $n=10$ per group) was measured and normalized to that of $P a x 7 C E /+$ heterozygous mice without tumors (set at $100 \%$ ). (C) Cross-sectional area measurements in LLC tumor-bearing Pax ${ }^{C E /+}$ and Pax7CE/f mice from B. (D) Cross-sectional area measurements of muscles from C-26 mice treated with systemic delivery of control siRNA or siRNA targeting Pax7 ( $n=5$ per group). (E) TA, GAST, and QUAD mass, measured from tumor-bearing mice lacking $(n=7)$ or expressing $(n=10)$ DTA under the control of the Pax7-Cre ${ }^{E R}$ locus. (F) Western blots performed on GAST from mice in $\mathbf{C}$ and $\mathbf{D}$, probing for MyoD and myogenin. Blots were reprobed for $\alpha$-tubulin. (G) GAST mass from C-26 $\mathrm{MyoD}^{+/+}$and $M y o D^{-/-}$mice ( $n=10$ per group) was measured and normalized to that from age- and genotype-matched nontumor control mice (set at $100 \%$ ). (H) Muscles of C-26 mice were injected with control or MyoD-expressing AAV ( $n=5$ per group); after 3 weeks of virus expression, muscle mass was measured and normalized to that from nontumor mice. ${ }^{*} P<0.05,{ }^{* \star} P<0.01$.

cess of neonatal muscle growth that relies on dividing myoblasts undergoing fusion with surrounding myofibers (43). Using a retroviral expression system ( $\mathrm{pBRIT}$ ) to target proliferating cells, we observed that Pax7 enhancement in myoblasts led to significant atrophy (Figure 5A and Supplemental Figure 7, C and D), a result that was repeated with a separate Pax7-pHIT retroviral system (Supplemental Figure 7, E and F). Thus, we concluded that Pax7 is sufficient to induce wasting.

Next, we performed loss-of-function experiments. C-26 tumors were administered to mice lacking 1 functional copy of $\operatorname{Pax} 7$ (a similar analysis was not feasible with $\operatorname{Pax}^{-1-}$ mice, which are runted shortly after birth and fail to thrive; ref. 23). Tumor-bearing Pax $7^{+/-}$ mice retained considerably more muscle mass than did $\mathrm{Pax} 7^{+/+}$mice $(P=0.002$; Supplemental Figure $8 \mathrm{~A})$. Consistent with this rescue, myofiber cross-sectional area from $\mathrm{Pax} 7^{+/-}$mice was also on average 22.6\% larger than $\mathrm{Pax} 7^{+/+}$mice $(P=0.03$; Supplemental Figure $8 \mathrm{~B})$. We confirmed that Pax7 haploinsufficiency did not affect tumor growth, nor did we observe significant differences in muscle mass or fiber size between non-tumor-bearing $\mathrm{Pax} 7^{+/+}$and $\mathrm{Pax} 7^{+/-}$ littermates (Supplemental Figure 8C). Similar effects were seen with $\mathrm{Pax}^{+/-}$mice bearing LLC tumors (Supplemental Figure 8D). To validate these findings, TA muscles from control and tumorbearing mice were transduced with a retrovirus expressing a shRNA
(pSuper) targeted against Pax7. This shRNA was effective at knocking down Pax7 (Supplemental Figure 9A), and, similar to $\mathrm{Pax} 7^{+/-}$ mice, muscle size was increased by $17 \%(P=0.025$; Supplemental Figure 9B). To confirm that these effects of Pax7 were derived from muscle progenitors, we used $\operatorname{Pax} 7^{\mathrm{CE} / f}$ mice, in which $\operatorname{Pax} 7$ was conditionally inactivated in stem cells by tamoxifen-induced Cre expression under the control of the Pax7 locus (44). Compared with tumor-bearing $\mathrm{Pax} 7^{\mathrm{CE} / \mathrm{+}}$ mice, containing 1 functional copy of $P a x 7$, the $P a x 7^{C E / f}$ mice lacking both copies retained significantly more muscle mass ( $9.8 \%$ increase; $P=0.02$; Figure $5 \mathrm{~B}$ ) and fiber size (25.5\% increase; $P=0.003$; Figure $5 C$ ). Taken together, these results strongly support the notion that sustained Pax7 expression in muscle progenitor cells is both required and sufficient to promote cancer-induced muscle wasting. To the best of our knowledge, this is the first report implicating the deregulation of Pax7 as a causal factor in any type of muscle pathology.

Based on these findings, we also tested Pax7 as a therapeutic target. Micro-osmotic pumps were implanted dorsally and used to systemically deliver a continuous, slow-release dose of siRNA against $\operatorname{Pax} 7$ for 1 week to tumor-bearing mice. Western blotting confirmed that Pax7 was effectively downregulated in hindlimb muscles (Supplemental Figure 9C); moreover, muscle fiber size increased $33.1 \%$ compared with control $(P=0.008$; Figure 5D). 
A

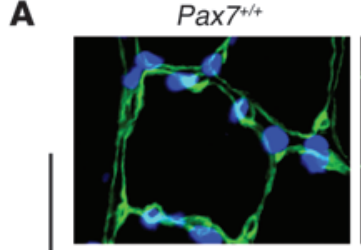

$\operatorname{Pax} 7^{\mathrm{CE} /+}$

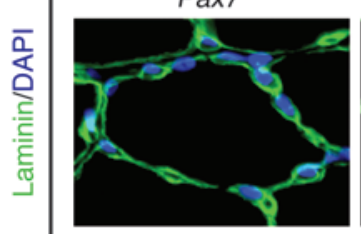

siRNA control

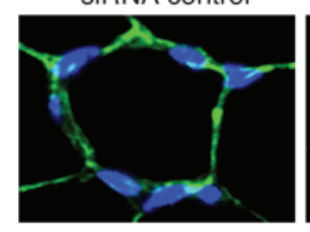

$P a x 7^{+/-}$

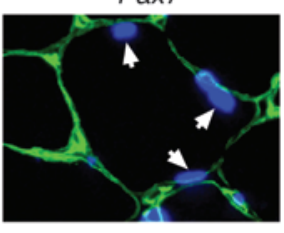

Pax7 CEA

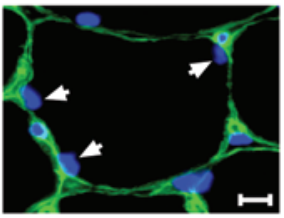

siRNA Pax7

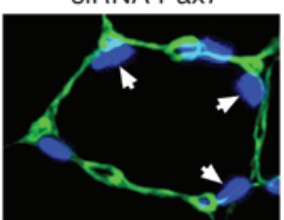

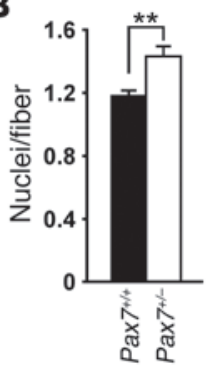

D

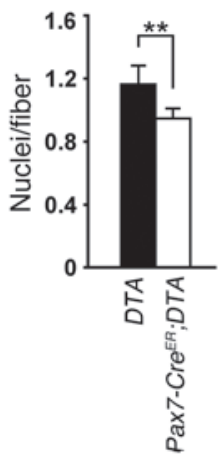

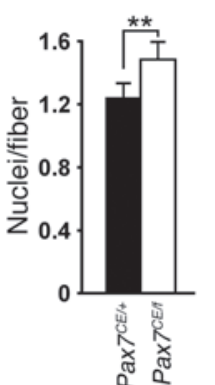

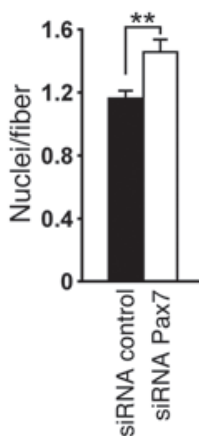

E

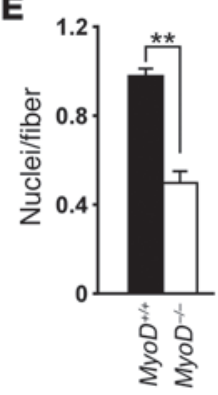

C

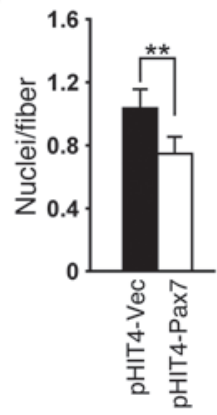

G
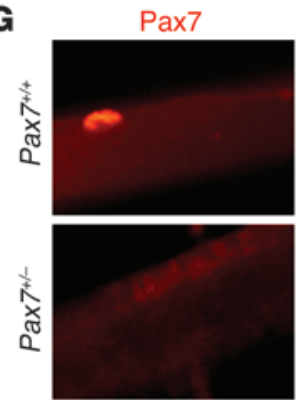

BrdU
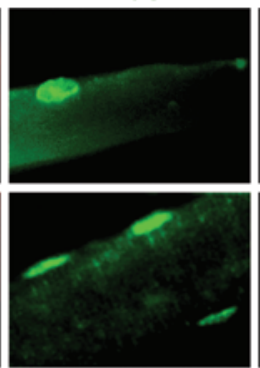
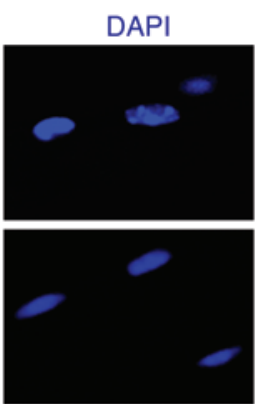
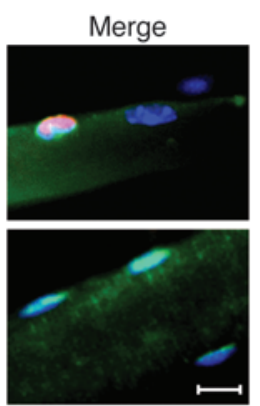

H

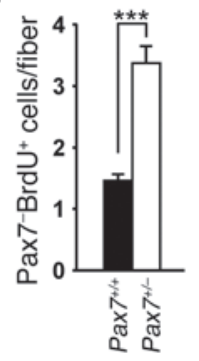

\section{Figure 6}

Deregulation of Pax7 promotes wasting in cancer by inhibiting regeneration through impairment of myogenic cell fusion. (A) Cross-sections prepared from muscles of tumor-bearing $\mathrm{Pax} 7^{+/+}$and $\mathrm{Pax} 7^{+/-}$mice, mice treated with control or siRNA against Pax7, or mice with conditional Pax7 deletion were immunostained with $\alpha$-laminin and DAPI. Arrows denote sublaminar nuclei. (B) Quantitation of sublaminar nuclei from A. At least 3,000 fibers were counted per condition. (C-F) Sublaminar nuclei per fiber from neonatal muscles injected with pHIT4 vector or Pax7 retroviruses (C); muscles in which Pax7+ progenitors were eliminated by DTA (D); $M y o D^{+/+}$and $M y o D^{-/-}$muscles (E); and muscles injected with control or MyoD-expressing AAV (F). At least 3,000 fibers were counted per condition. (G) Tumor-bearing Pax $7^{+/+}$and $P$ ax $7^{+/-}$mice were injected with BrdU, and muscle fibers were immunostained for Pax7, BrdU, and DAPI. Merged images are shown to denote specificity. (H) Quantitation of Pax7-BrdU+ myonuclei per fiber from muscles in G. Scale bars: $10 \mu \mathrm{m}(\mathbf{A}) ; 25 \mu \mathrm{m}(\mathbf{G}) .{ }^{* *} P<0.01,{ }^{* * *} P<0.001$.

Thus, targeting Pax7 may be useful as a therapeutic intervention for cancer cachexia.

Pax7 causes wasting in cancer by inhibiting myoblast fusion. Next, we determined whether Pax7 in cachexia is connected to the block in differentiation. We reasoned that if inhibiting differentiation contributes to cachexia, then creating a more profound loss in regeneration potential should further exacerbate the wasting process. To this end, we conditionally depleted Pax $7^{+}$cells by expressing diphtheria toxin (DTA) from the Rosa26-eGFP-DTA allele (45) under the control of Pax7-Cre $e^{E R}$. Tamoxifen was administered to Pax7-Cre ${ }^{E R}$;Rosa26-eGFP-DTA mice after C-26 tumor cell injection, at a time when both satellite cells and interstitial Sca $1^{+} \mathrm{CD} 34^{+}$cells commit to a myogenic lineage. Compared with cachectic mice not treated with tamoxifen, depletion of $\mathrm{Pax}^{+}$cells accentuated muscle loss (Figure 5E and Supplemental Figure 10A). Such results strongly supported the notion that impaired differentiation of $\mathrm{Pax} 7^{+}$cells is not simply a consequence of cancer, but a contributing factor in the wasting state.

In cultured myoblasts, Pax7 inhibits differentiation through suppression of MyoD and myogenin (42). Since we found that these factors were deregulated in cachectic muscle (Figure 2E), we examined whether this is linked to Pax7. Depletion of Pax7 by specific Cre recombination or siRNA delivery led to concomitant increases in $\mathrm{MyoD}$ and myogenin (Figure 5F and Supplemental Figure 10, 

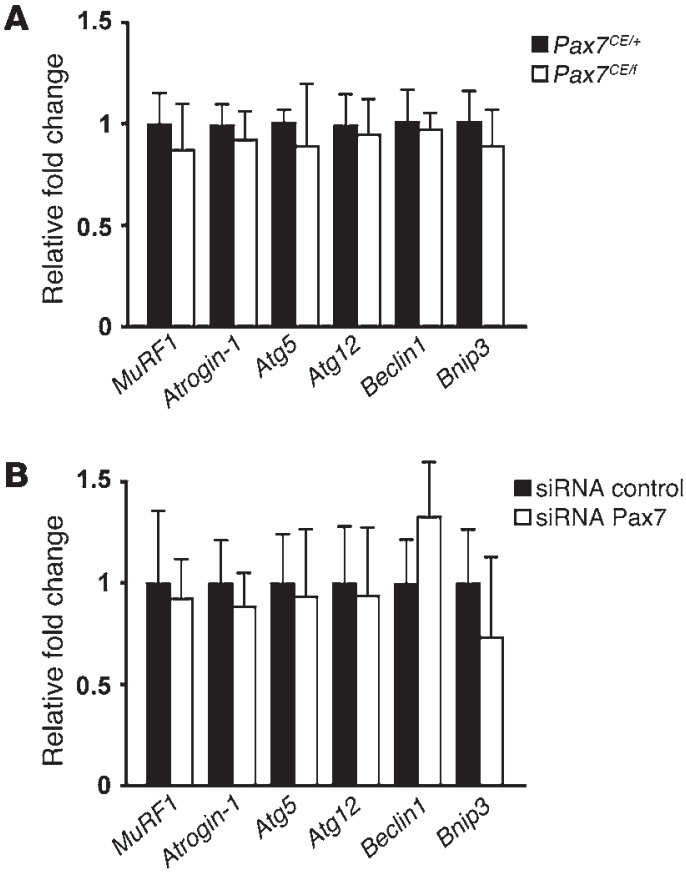

B and C). Furthermore, we reasoned that if Pax7 causes cachexia by inhibiting MyoD, then $M y o D^{-/-}$mice (46) should show greater tumor-induced muscle loss. Indeed, C-26 tumors in $\mathrm{MyoD}^{-1-}$ mice accentuated the decline relative to controls in muscle mass $(21.2 \%$; $P=0.003$; Figure 5G) and fiber size (51.8\%; $P=0.0004$; Supplemental Figure 10D). Similar findings were obtained in $\mathrm{MyoD}^{-/-}$mice bearing LLC tumors (Supplemental Figure 10E). To validate this regulation, we performed the converse experiment, expressing MyoD in cachectic muscles by AAV delivery. These conditions demonstrated successful restoration of muscle mass (10.5\% increase vs. control; $P=0.03$; Figure $5 \mathrm{H})$ and fiber size $(22.4 \%$ increase vs. control; $P=0.02$; Supplemental Figure 10F). As expected, MyoD rescue did not affect levels of Pax7, which acts upstream, but noticeably increased downstream expression of myogenin (Supplemental Figure 10G). These data suggested that Pax7 induces wasting by negatively acting on MyoD and myogenin.

Because myoblast fusion represents the last step in the differentiation program, we assessed whether Pax7 affected fusion in cachexia by scoring for $\mathrm{DAPI}^{+}$nuclei within myofibers. There was a significant increase in sublaminar myonuclei in muscles from Pax $7^{+/-}$mice $(P=0.0004$; Figure $6, \mathrm{~A}$ and $\mathrm{B})$. Similar results were obtained in muscles with progenitor cells conditionally lacking $\operatorname{Pax} 7(P=0.002)$ as well as in muscles from mice systemically treated with Pax7 siRNA ( $P=0.004$; Figure 6 , A and B). Conversely, sublaminar $\mathrm{DAPI}^{+}$nuclei were reduced when muscle wasting was accentuated by overexpressing Pax7 or by depleting Pax $7^{+}$cells with DTA (Figure 6, C and D). Impaired regeneration by Pax7 was dependent on $\mathrm{MyoD}$, since sublaminar nuclei were also reduced in tumor-bearing $\mathrm{MyoD}^{-/-}$mice $(P=0.002$; Figure $6 \mathrm{E})$, but increased in MyoD-overexpressing muscles $(P=0.008$; Figure $6 \mathrm{~F})$.

To examine more carefully whether increases in myonuclei from Pax7 knockdown are linked to myoblast fusion, we repeated tumor studies in $\mathrm{Pax}^{+/+}$and $\mathrm{Pax} 7^{+/-}$mice treated with BrdU to track myoblasts. Consistent with the above results, we found an appreciably higher number of sublaminar $\mathrm{Pax} 7^{-} \mathrm{BrdU}^{+}$myonuclei from $\mathrm{Pax} 7^{+/}$

\section{Figure 7}

Pax7-mediated muscle wasting in cancer is independent of proteasome and autophagy pathways. Muscles were isolated from tumorbearing mice either conditionally deleted for Pax7 in Pax $7^{+}$cells $(\mathbf{A})$ or systemically treated with siRNA targeting $\operatorname{Pax} 7(\mathbf{B})$, and RNA was analyzed by qRT-PCR probing for genes regulated in the ubiquitin proteasome (Atrogin-1 and MuRF1) and autophagy (Atg5, Atg12, Beclin1, and Bnip3) pathways.

mice (Figure 6, G and $\mathrm{H}$ ). This implies that $\mathrm{BrdU}^{+}$myoblasts from the interstitium of $\mathrm{Pax}^{+/-}$mice fused with myofibers, consequently downregulating Pax7 due to completion of their regenerative program. A similar phenomenon occurred when tumors were surgically removed from C-26 mice (Supplemental Figure 11, A and B), which, as shown earlier, rescued fusion and muscle atrophy (Figure 4, K and L). Thus, Pax7 regulates muscle wasting in cancer by inhibiting MyoD and myogenin that in turn restricts myoblast fusion to damaged myofibers in order to complete regeneration.

Notably, although the ubiquitin-dependent proteasome and autophagy systems are key regulators of cachexia (47-49), we observed that attenuating cachexia by reducing Pax7 had no effect on expression levels of E3 ubiquitin ligase or autophagy genes (Figure 7, A and B). In addition, although mediators of the protein synthetic pathway were paradoxically induced, as previously shown (18), no significant changes in Akt or mTOR activation occurred in cachectic muscles when Pax7 was conditionally depleted from myogenic progenitors (Supplemental Figure 12). These findings showed that the mechanism by which Pax7 promotes wasting in cancer is independent from those proteolytic pathways acting intrinsic to the myofiber.

$N F-\kappa B$ activation in myogenic progenitors regulates Pax 7 dysfunction and wasting. Finally, we sought to determine what factors act upstream of Pax7 in cachexia. Since our above findings supported the concept that wasting derives from tumor-associated circulating factors, we tested whether cachectic serum is sufficient to induce Pax7. Incubation of $\mathrm{C} 2 \mathrm{C} 12$ myoblasts with serum from cachectic mice $(n=7)$ increased Pax7 levels $148 \%$ over those of noncachectic mice $(n=3)(P=0.016$; Figure 8A). This activity was tumor derived, since serum from tumor-resected mice was considerably less effective at inducing Pax7 (Supplemental Figure 13A). Importantly, Pax7 was also elevated by $174 \%$ when C2C12 cells were incubated with serum from $P C$ patients with cachexia $(P=0.018$; Figure $8 B)$. Of 12 patients with $>5 \%$ weight loss, 10 stimulated Pax7 (Supplemental Table 1). Thus, Pax7 deregulation in cancer is likely under the control of circulating factors. Although inflammatory cytokines are associated with cachexia (50), we were unable to recapitulate the effect of cachectic serum with cytokines (TNF- $\alpha$, IL-1 $\beta$, IL- 6 , and IFN- $\gamma$, either individually or administered as a cocktail), nor with C-26 or LLC conditioned media (data not shown). To obtain initial information on the Pax7-inducing factor, cachectic serum was heat inactivated, delipidated, or fractionated by size to obtain small peptides. In each condition, Pax7 induction was maintained (Figure 8C), which indicates that the circulating factor is unlikely to be a complement protein, lipid, or $<10-\mathrm{kDa}$ peptide.

Since NF-кB negatively regulates skeletal myogenesis $(51,52)$ and positively regulates muscle atrophy under various cachexia conditions $(53,54)$, we asked whether NF- $\mathrm{KB}$ regulates Pax7 in cancer. Cachectic serum induced NF- $\mathrm{KB}$ transcriptional activity in C2C12 cells (Supplemental Figure 13B). Based on this result, 
A

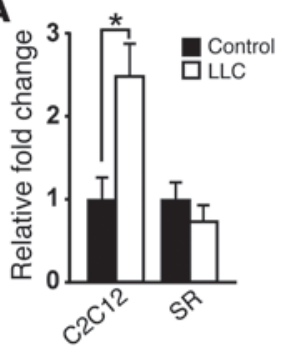

\section{B}

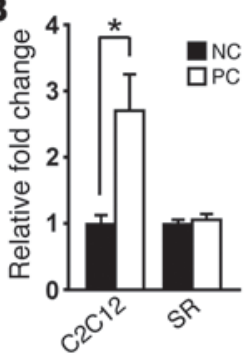

C

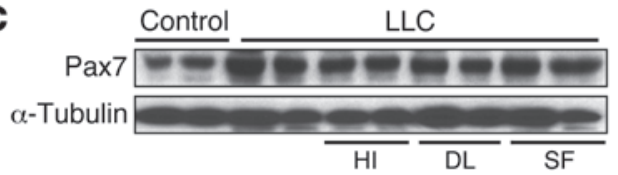

$\mathbf{E}$

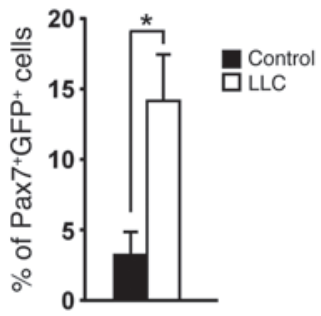

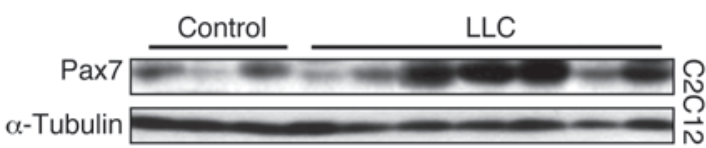

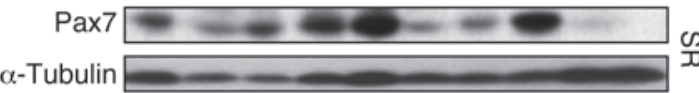

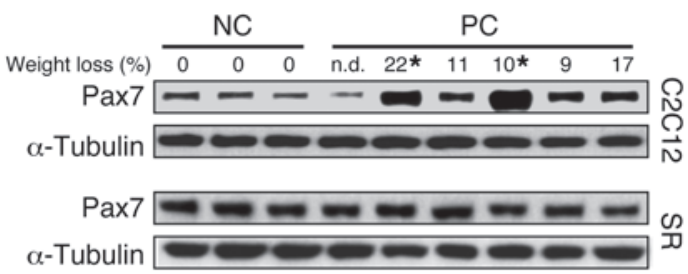
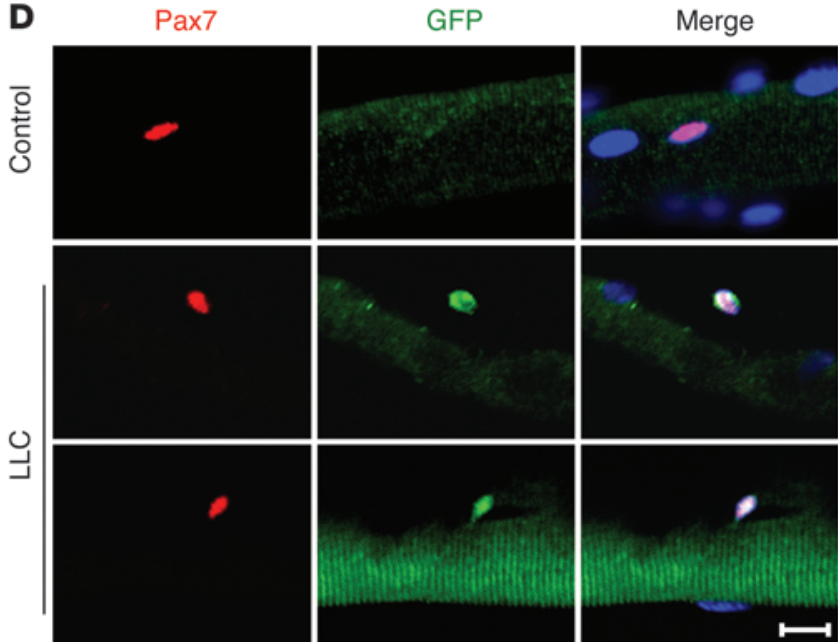

\section{Figure 8}

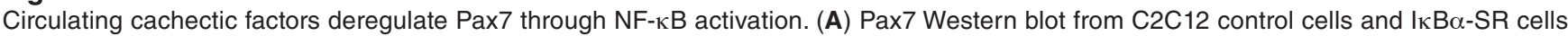
(SR) that were treated with $5 \%$ serum from control or LLC tumor-bearing mice. Quantitation of Pax7 normalized to $\alpha$-tubulin is also shown. (B) Similar to A, except serum was collected from NC and PC patients with weight loss as indicated (expressed as a percentage of the premorbid state). n.d., not determined. Asterisks denote samples from Figure 2D. Quantitation of Pax7 normalized to $\alpha$-tubulin is also shown. (C) Cachectic serum was heat inactivated $(\mathrm{HI})$, delipidated (DL), or size fractionated $<10 \mathrm{kDa}(\mathrm{SF})$ and used to treat $\mathrm{C} 2 \mathrm{C} 12 \mathrm{cells}$. Pax7 was examined by Western blot. (D) Fibers from control and LLC tumor-bearing mice were immunostained for Pax7 and GFP. (E) Quantitation of Pax7+GFP+ cells in D. Scale bars: $20 \mu \mathrm{m}(\mathbf{D}) .{ }^{*} P<0.05$.

serum experiments were repeated with myoblasts stably expressing

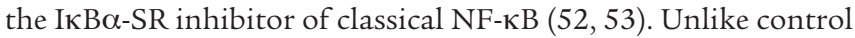
cells, Pax7 induction was blocked in IкB $\alpha$-SR cells when exposed to serum from either tumor-bearing mice or PC patients (Figure 8, $A$ and $B$ ), which supports the notion that cachectic factors require classical NF- $\mathrm{KB}$ to deregulate Pax7.

Next, we examined whether NF- $\mathrm{B}$ is activated in muscle progenitors in vivo. Using NF-кB GFP reporter mice (55), we found $\mathrm{NF}-\kappa \mathrm{B}$ activity to be increased in myofibers from tumor-bearing mice (Figure 8D), consistent with prior reports that NF- $\kappa B$ functions within myofibers to promote atrophy $(53,54)$. However, we also observed a more than a 3 -fold increase in $\mathrm{GFP}^{+} \mathrm{Pax} 7^{+}$cells (Fig- ure 8, D and $\mathrm{E}$ ), which suggests that NF-KB is activated in progenitor cells in cachectic muscle.

To determine whether NF- $\kappa$ B contributes to Pax7 deregulation and muscle wasting, we crossed constitutively kinase-active Rosa26-IKKß mice (56) with Pax7-Cre ${ }^{E R}$;Rosa26-Tomato mice. This allowed for selective activation of NF- $\kappa \mathrm{B}$ in $\mathrm{Pax} 7^{+}$cells, which could also be tracked by Tomato. Impressively, $I K K \beta$ induction led to an aggravated decline in muscle mass (Figure 9, A and C) and fiber size $(P=0.013$; Supplemental Figure $13, \mathrm{C}$ and $\mathrm{D})$ that correlated with increased Pax7 (Figure 9B). In addition, while some Tomato $^{+}$fibers could be seen in cachectic muscle, which indicated that fusion was not completely aborted, considerably less Tomato ${ }^{+}$ 
A

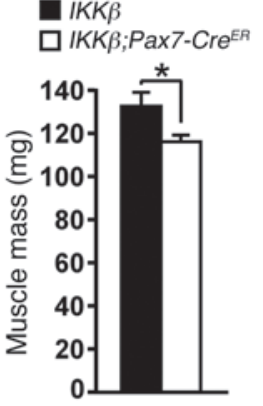

B

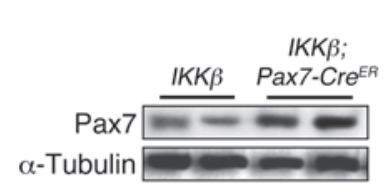

F

E

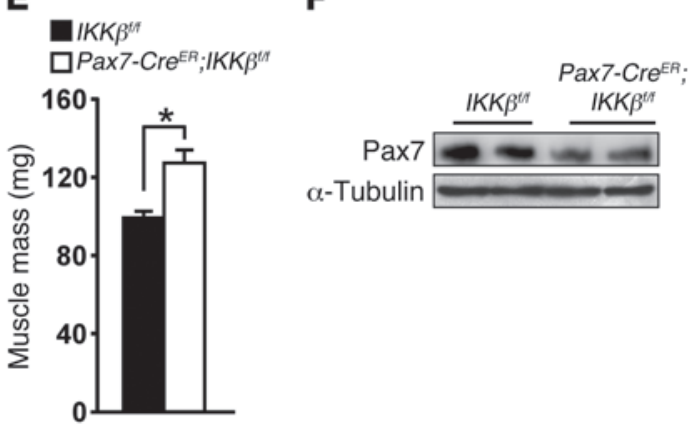

C

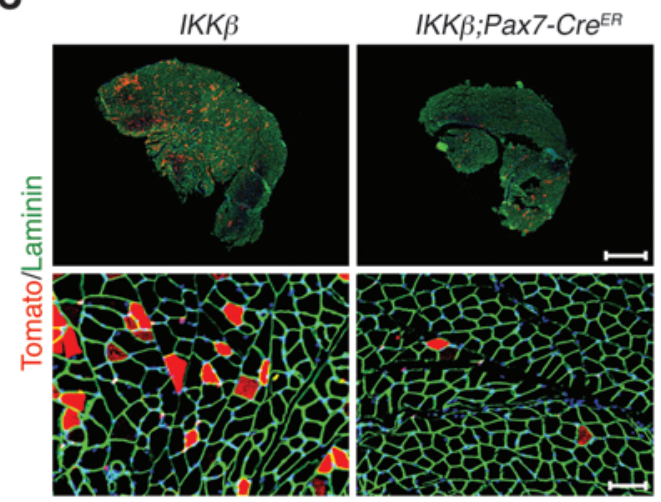

G

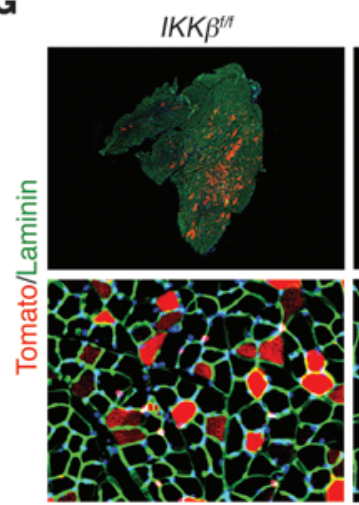

D
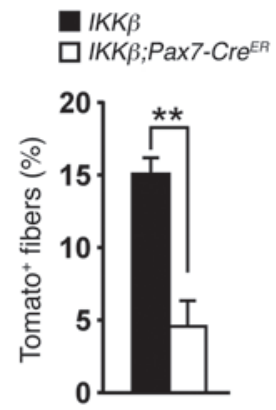

H

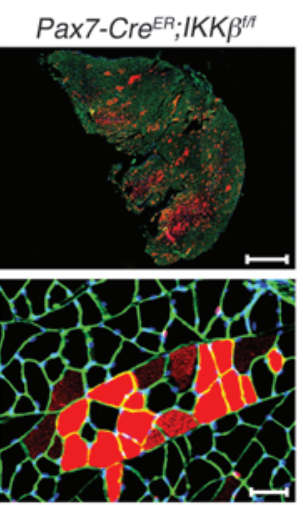

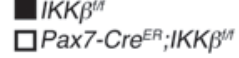

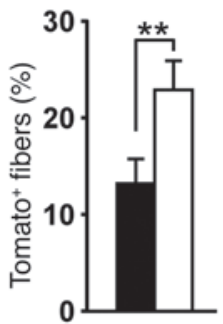

\section{Figure 9}

Pax7 deregulation and muscle wasting in cancer cachexia is dependent on NF-кB. (A) GAST mass from tumor-bearing control Rosa26-IKK $\beta$ $(n=15)$ or Pax7-CreER;Rosa26-IKK $(n=20)$ mice injected with tamoxifen. (B) Western blot for Pax7 from muscles in A. (C) Tomato cells from Rosa26-IKK $\beta$ and Pax7-Cre ${ }^{E R}$;Rosa26-IKK $\beta$ muscles probed for $\alpha$-laminin and DAPI (blue). Higher-magnification views are shown below. (D) Quantitation of Tomato+ cells from C. At least 15 sections and $9 \times 10^{5}$ fibers from 5 mice were counted for each group. (E) GAST mass from tumor-bearing control IKK $\beta^{f / f}(n=12)$ or Pax7-Cre ${ }^{E R ; I K K \beta^{f f f}}(n=18)$ mice injected with tamoxifen. $(\mathbf{F})$ Western blot of Pax7 from muscles in $\mathbf{E}$. (G) Tomato cells from $I K K \beta^{f / f}$ and Pax7-Cre $E R ; I K K \beta^{f f f}$ muscle sections immunostained with $\alpha$-laminin and DAPI (blue). Higher-magnification views are shown below. (H) Quantitation of Tomato+ fibers from G. At least 15 sections and $9 \times 10^{5}$ fibers from 3 mice were counted for each group. Scale bars: $0.5 \mathrm{~mm}$ (C and $\mathbf{G}$, top); $100 \mu \mathrm{m}\left(\mathbf{C}\right.$ and $\mathbf{G}$, bottom). ${ }^{*} P<0.05,{ }^{* \star} P<0.01$.

staining was measured in IKK $\beta$-expressing progenitors (Figure 9, $\mathrm{C}$ and D). Conversely, when NF- $\kappa \mathrm{B}$ was depleted in progenitors using conditionally mutant $I K K \beta^{f / f}$ mice (51) crossed with Pax7Cre ${ }^{E R}$;Rosa26-Tomato alleles, atrophy was spared (Figure 9, E and G, and Supplemental Figure 13, E and F; $P=0.008$ ). This rescue was associated with reduced levels of Pax7 (Figure 9F), concomitant with significant accumulation of Tomato $^{+}$fibers (Figure 9, G and $\mathrm{H})$, indicative of enhanced myoblast fusion and completed terminal differentiation. Collectively, these data firmly suggest that serum-derived cachectic factors signal through NF- $\mathrm{BB}$ in myogenic progenitors, to cause deregulation of Pax7 that in turn inhibits differentiation, thus promoting muscle wasting in cachexia.

Based on our above results, we asked whether NF-кB induction in stem cells is sufficient to drive wasting in a nontumor condition. Similar to conditions in which Pax7 was overexpressed in neonatal muscle (Figure 5A and Supplemental Figure 7, D-F), $\mathrm{NF}-\kappa \mathrm{B}$ induction in $\mathrm{Pax}^{+}$cells in neonates severely reduced muscle growth, while increasing Pax7. Compared with control mice, in which the Tomato reporter was observed in nearly $90 \%$ of the fibers, IKK $\beta$ expression in $\mathrm{Pax}^{+}$cells severely reduced fusion
(Supplemental Figure 13, G-I). These data support the notion that even in the absence of a tumor, induction of NF- $\mathrm{KB}$ in muscle progenitors acts upstream of Pax7 to impair differentiation and promote muscle atrophy.

\section{Discussion}

The present study was performed to provide perspective on the role of muscle injury and muscle progenitors in regulating atrophy during tumor progression. Our findings support the notion that satellite cells, along with other myogenic progenitor populations, become activated and enter into a regenerative program in response to muscle damage resulting from circulating cachectic factors. In this model, damaged myofibers from circulating tumor factors are not repaired, as myogenic differentiation is prematurely stalled and activated myogenic cells are unable to fuse to restore their growth (Figure 10). Although earlier reports described effects on the differentiation capacity of muscle progenitors in various atrophy models $(10,11,17)$, whether such dysregulation is sufficient to promote wasting had not been determined. Our findings strongly support, for the first time and on multiple levels, 


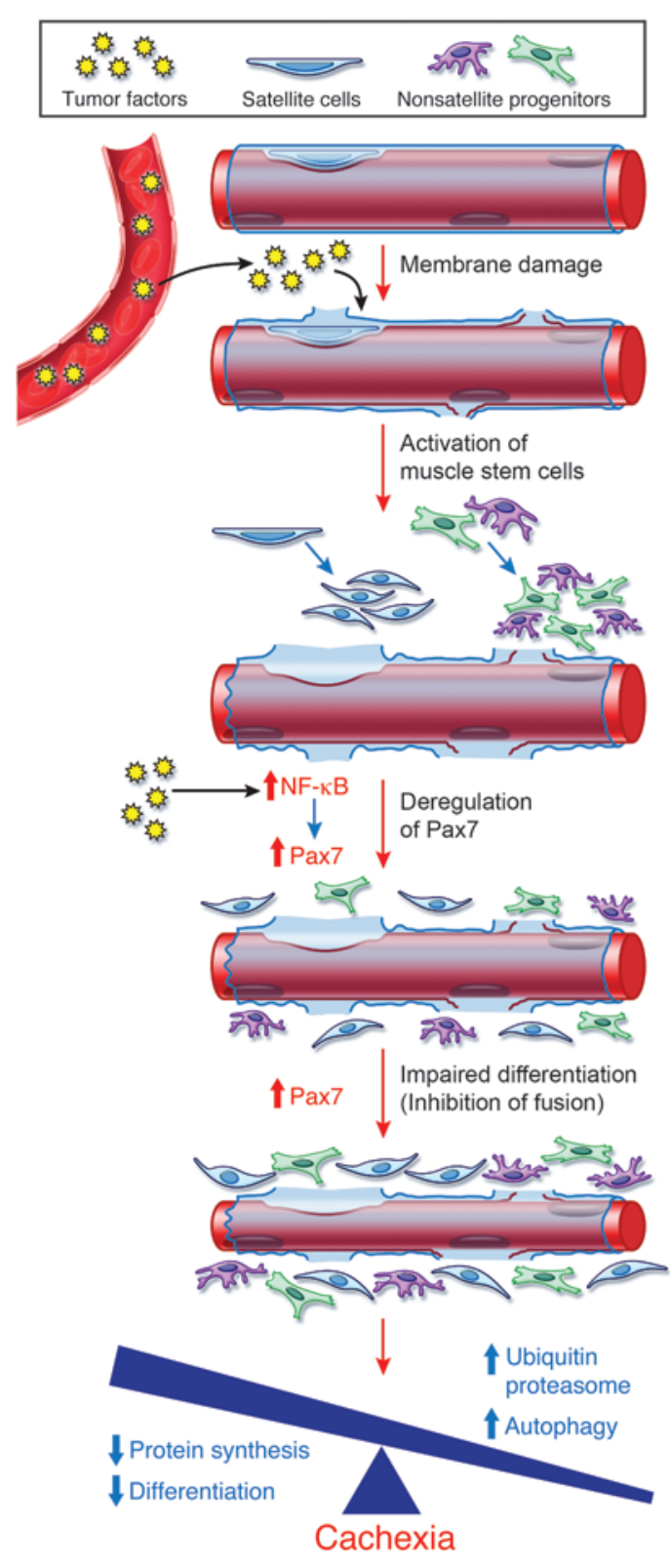

that differentiation is indeed impaired in cancer cachexia and that such impairment is a contributor in wasting. Although our data implied that the impairment in fusion derived from cell-autonomous events within myoblasts, we cannot rule out the possibility that the damage to the myofiber itself takes part in impairing differentiation. The accretion of myoblasts to preexisting myofibers is essential for hypertrophic muscle growth (57-59). Although evidence from an overloading model previously showed that hypertrophy is achieved even after satellite cells are depleted (60), it is possible that under such conditions, the protein synthetic machinery can become activated to compensate for the lack of myonuclear accretion. In our present studies, we showed that surgical tumor resection or conditional deletion of Pax7 in myogenic progenitors rescued muscle wasting, which coincided with an increase in myoblast fusion. However, under these conditions, we were unable to find alteration to the protein synthetic components Akt and mTOR (Supplemental Figure 12 and data not shown), which sup-

\section{Figure 10}

Involvement of the muscle microenvironment in cancer cachexia. Circulating tumor factors induce muscle membrane damage; in response, satellite cells and other myogenic progenitor populations are activated and commit to a myogenic program. Upon additional activation of classical NF- $\kappa B, P a x 7$ is deregulated, which impairs myogenic cell differentiation. Because of this stalled regeneration, committed myoblasts are unable to fuse and rescue damaged fibers. As a result, the loss of muscle repair in conjunction with reduced protein synthesis are unable to compensate against the procachectic activities of the ubiquitin proteasome and autophagy pathways, thus tipping the balance toward a cachectic state.

ports the concept that in cancer, loss of myonuclear accretion is sufficient to promote a procachectic state.

The exact nature of the circulating factors and mechanisms acting upstream of Pax7 warrant further investigation, but our current evidence points to at least one step involving NF-кB. Furthermore, we showed that NF-кB was activated in muscle progenitors during cachexia, leading to sustained Pax7 expression (Figure 10). In turn, a self-renewing signal in myogenic cells is likely propagated that results in their inability to progress through a differentiation program. Our findings that committed myogenic $\mathrm{Pax} 7^{+}$cells retained the stem cell markers Sca1 and CD34 as well as stromal markers that have previously been linked with muscle progenitors are consistent with this model, as well as with a prior demonstration that inhibition of Pax7 is required in order to progress through a myogenic program (61). Thus, activation of NF- $\kappa$ B leading to Pax7 appears crucial in directing proper muscle differentiation at different stages of development and under different environmental conditions. Whereas in fetal and juvenile muscles, Pax7 is required to sustain satellite cell renewal and regeneration in response to acute injury, its expression is thought to be dispensable in adult regenerating muscles (44). However, as shown in this study, sustained expression of Pax7 in neonatal or adult progenitors interferes with muscle growth and plays a pathological role in promoting muscle atrophy in the context of cancer. Similar to Pax7, activation of NF-кB in myoblasts has also been shown to inhibit MyoD synthesis (52). Therefore, the possibility exists that negative regulation of $\mathrm{MyoD}$ by NF- $\mathrm{KB}$ is mediated in part through Pax7.

Another concept revealed by our present findings was the involvement of nonsatellite cell progenitor populations in cancer cachexia. Multiple resident stem cells exist in skeletal muscle (7). By all accounts, these progenitors are nonmyogenic in resting muscle, but adopt a myogenic fate in response to acute injury signals. Unique to our study is the implication that nonsatellite progenitors contribute to muscle wasting by committing to myogenesis, but - like satellite cells - fail to complete differentiation in order to repair damaged fibers (Figure 10). Our data further revealed that PDGFR $\alpha^{+} \mathrm{Sca} 1^{+}$mesenchymal progenitor cells also possessed the capacity to convert to a myogenic lineage. Although such cells have recently been shown to facilitate acute muscle regeneration by interacting with innate immune cells (62), they themselves are considered to be nonmyogenic $(28,29)$. Based on our findings, we reason that conversion of PDGFR $\alpha^{+} \mathrm{Sca}^{+}$cells to a myogenic lineage is possible in the context of a chronic injury signal elicited by a specific tumor environment. The exact identity of these and other nonsatellite cell progenitors, as well as their specific contribution to muscle wasting in cancer, await further investigation. 
Cumulatively, we predict that impairment of muscle progenitors to complete differentiation exacerbates wasting in cancer by loss of a compensatory mechanism that serves to counteract the procachectic activities of the ubiquitin proteasome and autophagy pathways operating within the myofiber (Figure 10). This is analogous to a condition of insulin resistance whereby Akt signaling is inhibited and protein synthesis is reduced, leading to unregulated proteolysis. Whether such a similar mechanism underlies wasting in other atrophy conditions was not explored, but findings from denervation and disuse models argue against such a notion, since in these models satellite cell number decline (9-11) as opposed to increasing, as observed under a tumor burden. In addition, although our results demonstrated that myogenin was not induced during cancer cachexia, its expression was previously reported to be highly elevated in denervation and required for activation of the ubiquitin proteasome system (63). Similar to the idea raised by investigators in that study that myogenin does not function in the same manner in denervation as it does in starvationinduced atrophy, it is possible that the dysfunction of Pax7, and resulting impairment of muscle regeneration, is selective to cancer cachexia. Nevertheless, we envision that for therapeutic purposes, compounds that target Pax7 or NF-KB - and stimulate differentiation of myogenic cells - could be beneficial in modulating muscle loss in response to procachectic factors. Because impairment of the muscle regenerative program is thought to be a causal factor in muscular dystrophy, strategies to treat such degenerative disorders using compounds that stimulate myogenesis may also prove effective in restoring muscle mass in cancer patients.

\section{Methods}

Mice and cachexia measurement. Cachexia in C-26 and LLC models was previously described (64). $m d x$ mice (C57BL/10 ScSn DMD ${ }^{\mathrm{mdx}}$ ) were crossed into a CD2F1 up to 7 generations for C-26 injection. $n u / n u$ nude mice were purchased from Charles River Laboratories; Rosa26-Tomato reporter and GFP reporter mice were from Jackson Laboratories. $\mathrm{Pax} 7^{\mathrm{CE} /+}$ and $\mathrm{Pax} 7^{\mathrm{f} /+}$ mice (Jackson Laboratories) were crossed to obtain Pax $7^{C E / f}$ mice. To activate or deplete NF-кB in Pax $7^{+}$cells, Pax7-Cre ${ }^{E R}$ mice were crossed with Rosa26-IKK $\beta$ or $I K K \beta f / f$ mice. All genotypes were determined by PCR using tail DNA.

Patients. Rectus abdominis muscle biopsies were obtained at time of surgery from men and nonpregnant women $18-80$ years of age. Diagnosis was based on histological or unequivocal radiological or operative findings. No PC patients received preoperative chemotherapy. NC patients (healthy, weight-stable subjects undergoing minor surgical procedures, e.g., inguinal hernia repair) were included as controls. Body weight was measured with subjects in light clothing without shoes using a beam scale (Seca). Weight loss of PC subjects was determined at the time of surgery compared with preoperative stable body weight.

Electron microscopy, histology, and confocal microscopy. Muscles were fixed in $3 \%$ glutaraldehyde, then stained and sectioned for transmission EM
(Spirit Tecnai; FEI) as previously described (18). For histology, tissues were sectioned on a cryostat (Leica) and processed for H\&E staining or immunofluorescence as described previously (18), and images were captured with Carl Zeiss Axioskop fluorescence microscope (Axioskop 40; Carl Zeiss Inc.) or single-photon Olympus FV1000 confocal microscope.

Primers and antibodies. See Supplemental Table 2 for primers for RT-PCR and real-time RT-PCR. See Supplemental Table 3 for primary and secondary antibodies for Western blotting. See Supplemental Table 4 for primary and secondary antibodies for immunofluorescence as well as antibodies for flow cytometry.

Statistics. All quantitative data are represented as mean or as mean \pm SEM. Analysis was performed between different groups using 2-tailed Student's $t$ test. A $P$ value less than 0.05 was considered significant; a $P$ value less than 0.01 was considered highly significant.

Study approval. All animal protocols were approved and conducted in accordance with University Laboratory Animal Resources of The Ohio State University and Institutional Animal Care and Use Committee regulations. Studies involving human subjects were done in accordance with the Institutional Review Boards of The Ohio State University, and patients provided informed consent.

\section{Acknowledgments}

We are grateful to $\mathrm{Z}$. Li for the use of desmin-nLacZ transgenic mice, C. Jobin for NF- $\kappa B$ reporter mice, and M. Karin for IKK $\beta$ mutant mice. We thank C. Penton for technical assistance in FACS sorting, W. Kline in the Guttridge laboratory for assistance in myofiber quantification analysis, M. Freitas and M. Belury for helpful discussions, and the other members of the laboratory for their support throughout the course of this study. This work was supported by Emergence 2011 and AFM 2012-0773 to D. Coletti; by NIH grants K01CA097953, R01CA098466, and R03CA124692 to D.C. Guttridge; and by CCTS (UL1TR000090) awards to F. Montanaro and D.C. Guttridge.

Received for publication December 26, 2012, and accepted in revised form August 6, 2013.

Address correspondence to: Denis C. Guttridge, $460 \mathrm{~W} .12^{\text {th }}$ Avenue, The Ohio State University College of Medicine, Columbus, Ohio 43210, USA. Phone: 614.688.3137; Fax: 614.688.4006; E-mail: denis.guttridge@osumc.edu.

Emanuele Berardi's present address is: Translational Cardiomyology Lab, Department of Development and Regeneration, Stem Cell Institute Leuven, Embryo and Stem Cell Biology, KU Leuven, Leuven, Belgium.

Dario Coletti's present address is: University Pierre et Marie Curie, Paris 06, Paris, France.
1. Tisdale MJ. Cachexia in cancer patients. Nat Rev Cancer. 2002;2(11):862-871.

2. Andreyev HJ, Norman AR, Oates J, Cunningham D. Why do patients with weight loss have a worse outcome when undergoing chemotherapy for gastrointestinal malignancies? Eur J Cancer. 1998; 34(4):503-509.

3. Fearon KC, Glass DJ, Guttridge DC. Cancer cachexia: mediators, signaling, and metabolic pathways. Cell Metab. 2012;16(2):153-166.

4. Tisdale MJ. Tumor-host interactions. J Cell Biochem. 2004;93(5):871-877.

5. Siegel R, Naishadham D, Jemal A. Cancer statistics,
2012. CA Cancer J Clin. 2012;62(1):10-29.

6. Glass DJ. Signaling pathways perturbing muscle mass. Curr Opin Clin Nutr Metab Care. 2010; 13(3):225-229.

7. Pannerec A, Marazzi G, Sassoon D. Stem cells in the hood: the skeletal muscle niche. Trends Mol Med. 2012;18(10):599-606.

8. Mauro A. Satellite cell of skeletal muscle fibers. J Biophys Biochem Cytol. 1961;9(2):493-495.

9. Doppler K, Mittelbronn M, Bornemann A. Myogenesis in human denervated muscle biopsies. Muscle Nerve. 2008;37(1):79-83.

10. Viguie CA, Lu DX, Huang SK, Rengen H, Carlson
BM. Quantitative study of the effects of long-term denervation on the extensor digitorum longus muscle of the rat. Anat Rec. 1997;248(3):346-354.

11. Mitchell PO, Pavlath GK. Skeletal muscle atrophy leads to loss and dysfunction of muscle precursor cells. Am J Physiol Cell Physiol. 2004; 287(6):C1753-C1762.

12. Langen RC, Schols AM, Kelders MC, van der Velden JL, Wouters EF, Janssen-Heininger YM. Muscle wasting and impaired muscle regeneration in a murine model of chronic pulmonary inflammation. Am J Respir Cell Mol Biol. 2006;35(6):689-696.

13. Penna F, Costamagna D, Fanzani A, Bonelli G, Bac- 
cino FM, Costelli P. Muscle wasting and impaired myogenesis in tumor bearing mice are prevented by ERK inhibition. PloS One. 2010;5(10):e13604.

14. Wu X, Walters TJ, Rathbone CR. Skeletal muscle satellite cell activation following cutaneous burn in rats. Burns. 2013;39(4):736-744.

15. Zhang L, Wang XH, Wang H, Du J, Mitch WE. Satellite cell dysfunction and impaired IGF-1 signaling cause CKD-induced muscle atrophy. J Am Soc Nephrol. 2010;21(3):419-427.

16. Coletti D, Moresi V, Adamo S, Molinaro M, Sassoon $\mathrm{D}$. Tumor necrosis factor-alpha gene transfer induces cachexia and inhibits muscle regeneration. Genesis. 2005;43(3):120-128.

17. Schwarzkopf M, Coletti D, Sassoon D, Marazzi G. Muscle cachexia is regulated by a p53-PW1/ Peg3-dependent pathway. Genes Dev. 2006; 20(24):3440-3452

18. Acharyya S, et al. Dystrophin glycoprotein complex dysfunction: a regulatory link between muscular dystrophy and cancer cachexia. Cancer Cell. 2005;8(5):421-432.

19. Karpati G, Carpenter S. Pathology Of Skeletal Muscle. New York, New York, USA: Oxford University Press; 2001.

20. Wang XY, Rudnicki MA. Satellite cells, the engines of muscle repair. Nat Rev Mol Cell Biol. 2012;13(2):127-133.

21. Deconinck AE, et al. Utrophin-dystrophin-deficient mice as a model for Duchenne muscular dystrophy. Cell. 1997;90(4):717-727.

22. Hawke TJ, Garry DJ. Myogenic satellite cells: physiology to molecular biology. J Appl Physiol. 2001;91(2):534-551.

23. Seale P, Sabourin LA, Girgis-Gabardo A, Mansouri A, Gruss P, Rudnicki MA. Pax7 is required for the specification of myogenic satellite cells. Cell. 2000;102(6):777-786

24. Dellavalle A, et al. Pericytes resident in postnatal skeletal muscle differentiate into muscle fibres and generate satellite cells. Nat Commun. 2011;2:499.

25. Mitchell KJ, et al. Identification and characterization of a non-satellite cell muscle resident progenitor during postnatal development. Nat Cell Biol. 2010;12(3):257-266

26. Qu-Petersen Z, et al. Identification of a novel population of muscle stem cells in mice: potential for muscle regeneration. JCell Biol. 2002;157(5):851-864.

27. Peault B, et al. Stem and progenitor cells in skeletal muscle development, maintenance, and therapy. Mol Ther. 2007;15(5):867-877.

28. Joe AW, et al. Muscle injury activates resident fibro/ adipogenic progenitors that facilitate myogenesis. Nat Cell Biol. 2010;12(2):153-163.

29. Uezumi A, Fukada S, Yamamoto N, Takeda S, Tsuchida K. Mesenchymal progenitors distinct from satellite cells contribute to ectopic fat cell formation in skeletal muscle. Nat Cell Biol. 2010;12(2):143-152.

30. Bosnakovski D, et al. Prospective isolation of skeletal muscle stem cells with a Pax7 reporter. Stem
Cells. 2008;26(12):3194-3204.

31. Mitchell PO, et al. Sca-1 negatively regulates proliferation and differentiation of muscle cells. Dev Biol. $2005 ; 283: 240-252$

32. Tanaka KK, Hall JK, Troy AA, Cornelison DD, Majka SM, Olwin BB. Syndecan-4-expressing muscle progenitor cells in the SP engraft as satellite cells during muscle regeneration. Cell Stem Cell. 2009;4(3):217-225.

33. Nishijo K, et al. Biomarker system for studying muscle, stem cells, and cancer in vivo. FASEB J. 2009; 23(8):2681-2690.

34. Dellavalle A, et al. Pericytes of human skeletal muscle are myogenic precursors distinct from satellite cells. Nat Cell Biol. 2007;9(3):255-267.

35. Beauchamp JR, et al. Expression of CD34 and Myf5 defines the majority of quiescent adult skeletal muscle satellite cells. J Cell Biol. 2000;151(6):1221-1234.

36. Ieronimakis N, Balasundaram G, Rainey S, Srirangam $\mathrm{K}$, Yablonka-Reuveni $Z$, Reyes $\mathrm{M}$. Absence of CD34 on murine skeletal muscle satellite cells marks a reversible state of activation during acute injury. PLoS One. 2010;5(6):e10920.

37. Hasty P, et al. Muscle deficiency and neonatal death in mice with a targeted mutation in the myogenin gene. Nature. 1993;364(6437):501-506.

38. Megeney LA, Kablar B, Garrett K, Anderson JE, Rudnicki MA. MyoD is required for myogenic stem cell function in adult skeletal muscle. Genes Dev. 1996;10(10):1173-1183.

39. Nabeshima Y, Hanaoka K, Hayasaka M, Esumi E, Li $\mathrm{S}$, Nonaka I. Myogenin gene disruption results in perinatal lethality because of severe muscle defect. Nature. 1993;364(6437):532-535.

40. Blake DJ, Weir A, Newey SE, Davies KE. Function and genetics of dystrophin and dystrophin-related proteins in muscle. Physiol Rev. 2002;82(2):291-329.

41. Li Z, Marchand P, Humbert J, Babinet C, Paulin D. Desmin sequence elements regulating skeletal muscle-specific expression in transgenic mice. Development. 1993;117(3):947-959.

42. Olguin HC, Olwin BB. Pax-7 up-regulation inhibits myogenesis and cell cycle progression in satellite cells: a potential mechanism for self-renewal. Dev Biol. 2004;275(2):375-388

43. White RB, Bierinx AS, Gnocchi VF, Zammit PS. Dynamics of muscle fibre growth during postnatal mouse development. BMC Dev Biol. 2010;10:21-32.

44. Lepper C, Conway SJ, Fan CM. Adult satellite cells and embryonic muscle progenitors have distinct genetic requirements. Nature. 2009;460(7255):627-631.

45. Ivanova A, Signore M, Caro N, Greene ND, Copp AJ, Martinez-Barbera JP. In vivo genetic ablation by Cre-mediated expression of diphtheria toxin fragment A. Genesis. 2005;43(3):129-135.

46. Rudnicki MA, Braun T, Hinuma S, Jaenisch R. Inactivation of MyoD in mice leads to up-regulation of the myogenic HLH gene Myf-5 and results in apparently normal muscle development. Cell. 1992; 71(3):383-390.
47. Bodine SC, et al. Identification of ubiquitin ligases required for skeletal muscle atrophy. Science. 2001;294(5547):1704-1708.

48. Gomes MD, Lecker SH, Jagoe RT, Navon A, Goldberg AL. Atrogin-1, a muscle-specific F-box protein highly expressed during muscle atrophy. Proc Natl Acad Sci U S A. 2001;98(25):14440-14445.

49. Mammucari $\mathrm{C}$, et al. FoxO3 controls autophagy in skeletal muscle in vivo. Cell Metab. 2007;6(6):458-471.

50. Argilés JM, López-Soriano FJ. The role of cytokines in cancer cachexia. Med Res Rev. 1999;19(3):223-248.

51. Acharyya $S$, et al. Interplay of IKK/NF-kappaB signaling in macrophages and myofibers promotes muscle degeneration in Duchenne muscular dystrophy. J Clin Invest. 2007;117(4):889-901.

52. Guttridge DC, Mayo MW, Madrid LV, Wang CY, Baldwin AS. NF- $\mathrm{B}$-induced loss of MyoD messenger RNA: possible role in muscle decay and cachexia. Science. 2000;289(5488):2363-2366.

53. Cai $\mathrm{D}$, et al. IKK $\beta / \mathrm{NF}-\kappa \mathrm{B}$ activation causes severe muscle wasting in mice. Cell. 2004;119(2):285-298.

54. Mourkioti F, et al. Targeted ablation of IKK2 improves skeletal muscle strength, maintains mass, and promotes regeneration. J Clin Invest. 2006; 116(11):2945-2954

55. Dahlman JM, Bakkar N, He W, Guttridge DC. NF$\kappa \mathrm{B}$ functions in stromal fibroblasts to regulate early postnatal muscle development. J Biol Chem. 2010; 285(8):5479-5487.

56. Sasaki Y, et al. Canonical NF-kappaB activity, dispensable for B cell development, replaces BAFFreceptor signals and promotes $B$ cell proliferation upon activation. Immunity. 2006;24(6):729-739.

57. Guerci A, et al. Srf-dependent paracrine signals produced by myofibers control satellite cell-mediated skeletal muscle hypertrophy. Cell Metab. 2012; 15(1):25-37.

58. Horsley V, Jansen KM, Mills ST, Pavlath GK. IL-4 acts as a myoblast recruitment factor during mammalian muscle growth. Cell. 2003;113(4):483-494.

59. Serrano AL, Baeza-Raja B, Perdiguero E, Jardi M, Munoz-Canoves P. Interleukin- 6 is an essential regulator of satellite cell-mediated skeletal muscle hypertrophy. Cell Metab. 2008;7(1):33-44.

60. McCarthy JJ, et al. Effective fiber hypertrophy in satellite cell-depleted skeletal muscle. Development. 2011;138(17):3657-3666.

61. Palacios $\mathrm{D}$, et al. TNF/p $38 \alpha /$ polycomb signaling to Pax7 locus in satellite cells links inflammation to the epigenetic control of muscle regeneration. Cell Stem Cell. 2010;7(4):455-469.

62 . Heredia JE, et al. Type 2 innate signals stimulate fibro/adipogenic progenitors to facilitate muscle regeneration. Cell. 2013;153(2):376-388.

63. Moresi V, et al. Myogenin and class II HDACs control neurogenic muscle atrophy by inducing E3 ubiquitin ligases. Cell. 2010;143(1):35-45.

64 . Acharyya $\mathrm{S}$, et al. Cancer cachexia is regulated by selective targeting of skeletal muscle gene products. J Clin Invest. 2004;114(3):370-378. 